\title{
Spin-orbit effects in single-electron states in coupled quantum dots
}

\author{
Peter Stano ${ }^{1,2}$ and Jaroslav Fabian ${ }^{1,2}$ \\ ${ }^{1}$ Institute of Physics, Karl-Franzens University, Universitätsplatz, 5, 8010 Graz, Austria \\ ${ }^{2}$ Institute for Theoretical Physics, University of Regensburg, 93040 Regensburg, Germany \\ (Received 23 June 2005; revised manuscript received 19 August 2005; published 14 October 2005)
}

\begin{abstract}
Spin-orbit effects in single-electron states in laterally coupled quantum dots in the presence of a perpendicular magnetic field are studied by exact numerical diagonalization. Dresselhaus (linear and cubic) and Bychkov-Rashba spin-orbit couplings are included in a realistic model of confined dots based on GaAs. Group theoretical classification of quantum states with and without spin-orbit coupling is provided. Spin-orbit effects on the $g$ factor are rather weak. It is shown that the frequency of coherent oscillations (tunneling amplitude) in coupled dots is largely unaffected by spin-orbit effects due to symmetry requirements. The leading contributions to the frequency involves the cubic term of the Dresselhaus coupling. Spin-orbit coupling in the presence of a magnetic field leads to a spin-dependent tunneling amplitude, and thus to the possibility of spin to charge conversion, namely, spatial separation of spin by coherent oscillations in a uniform magnetic field. It is also shown that spin hot spots exist in coupled GaAs dots already at moderate magnetic fields, and that spin hot spots at zero magnetic field are due to the cubic Dresselhaus term only.
\end{abstract}

DOI: 10.1103/PhysRevB.72.155410

PACS number(s): 73.21.La, 71.70.Ej

\section{INTRODUCTION}

The possibility of tuning spin-orbit coupling ${ }^{1-3}$ in lowdimensional semiconductor electronic structures has stirred great interest in spin properties of lateral semiconductor electron systems in the presence of Dresselhaus ${ }^{4}$ and Bychkov-Rashba ${ }^{5,6}$ spin-orbit couplings. The former appears in low-dimensional systems lacking inversion symmetry in the bulk (such as zinc-blende semiconductors), the latter in low-dimensional structures with asymmetric confining potentials. The principal question is what spin and charge properties and to what degree can be affected and manipulated by this tuning? Such questions are of fundamental importance for spintronics. ${ }^{7}$

Electron spins in coupled quantum dot systems have been proposed to perform universal gating of quantum computers. ${ }^{8}$ The spin acts as a qubit and exchange coupling provides the physical realization of two-qubit gates. ${ }^{9,10}$ Another application of a controlled coupling between dots is spin entanglement distillation in which singlet and triplet states get spatially separated during adiabatic passage through trapped states. ${ }^{11}$ The understanding of spin-orbit effects in coupled dots is thus of great interest to quantum information processing.

Spin-orbit coupling provides a way for orbital degrees of freedom to influence spin states. As a result the spin dynamics is affected, making spin qubit operations more complex (it was shown, though, that two-qubit operations can be performed reliably even in the presence of spin-orbit interaction which leads to anisotropic exchange ${ }^{12,13}$ ). Furthermore, spinorbit coupling leads to spin decoherence and relaxation due to phonons, ${ }^{14-19}$ limiting the operation time. The impressive experimental progress in coherent oscillations in coupled dot systems, ${ }^{20-23}$ as well in spin dephasing and spin manipulation in single ${ }^{24,25}$ and double dots, ${ }^{26}$ provides additional strong impetus for investigating spin states in double dots. Spin-orbit effects on single dots have been already extensively investigated. ${ }^{27-38}$
In this paper we investigate the role of spin-orbit coupling, represented by the Dresselhaus (both linear and cubic) and Bychkov-Rashba terms, in spin and charge properties of two laterally coupled quantum dots based on GaAs materials parameters. We perform numerically exact calculations of the energy spectrum using the method of finite differences. We first study the general structure of the energy spectrum and the spin character of the states of the double dot system. We construct the group theoretical correlation diagram for the single and double dot states and indicate the possible transitions due to spin-orbit coupling. This group theoretical classification is used in combination with the Löwdin perturbation theory to explain analytically our numerical results. In particular, we show that while allowed by symmetry, the specific forms of the linear spin-orbit interactions do not lead to spin hot spots in the absence of magnetic field (spin hot spots are nominally degenerate states lifted by spin-orbit coupling ${ }^{39}$ ). Only the cubic Dresselhaus term gives spin hot spots. If identified experimentally, the strength of the cubic term can be detected.

We next focus on two important measurable parameters: electronic $g$ factor and tunneling amplitude. In single dots the variation of the effective $g$ factor with the strength of the spin-orbit interaction has been investigated earlier. ${ }^{29}$ The effect is not large, amounting to a fraction of a percent. Similar behavior is found for double dots. In our case of GaAs the contribution to the $g$ factor from spin-orbit coupling is typically about $1 \%$, due to the linear Dresselhaus term.

More exciting is the prospect of influencing coherent tunneling oscillations between the dots by modulating the spinorbit coupling strength. Two effects can appear: (i) the tunneling amplitude or frequency can be modulated by spinorbit coupling and (ii) the tunneling amplitude can be spin dependent. We show how a naive application of the perturbation theory leads to a misleading result that (i) is present in the second order in linear spin-orbit coupling strengths, giving rise to an effective tunneling Hamiltonian involving spinflip tunneling at zero magnetic field. Both numerical calcu- 
lations and an analytical argument, presented here, show that this is incorrect and that there is no correction to the tunneling Hamiltonian in the second order of linear spin-orbit terms. The dominant correction in the second order comes from the interference of linear and cubic Dresselhaus terms. We propose to use this criterion, that the corrections to linear terms vanish in the second order, to distinguish between single and double dots as far as spin-orbit coupling is concerned. Indeed, at very small and very large interdot couplings the states have a single dot character and the correction to energy due to linear spin-orbit terms depends on the interdot distance (except for the two lowest states which provide tunneling). We find that dots are "coupled" up to the interdot distance of about five single-dot confinement lengths.

In the presence of magnetic field the time reversal symmetry is broken. The presence of spin-orbit coupling then, in general, leads to a spin dependent tunneling amplitude. Spin up and spin down states will oscillate between the two dots with different frequencies (for our GaAs dots the relative difference of the frequencies is at the order of $0.1 \%$, but is higher in materials with larger spin-orbit coupling). This leads to a curious physical effect, namely, that of a spatial separation of different spin species. Indeed, starting with an electron localized on one dot, with a spin polarized in the plane (that is, a superposition of up and down spins), after a sequence of coherent oscillations the electron state is a superposition of spin up localized on one, and spin down localized on the other dot. A single charge measurement on one dot collapses the wave function to the corresponding spin state, realizing a spin to charge conversion. There exist several alternative schemes, ${ }^{40-43}$ some of them being pursued experimentally. ${ }^{24,25,44}$ We construct an effective, four state (two spin and two sites) tunneling Hamiltonian for the single-electron double dot system, which takes into effect the above results. Such a Hamiltonian should be useful for constructing realistic model theories of spin dephasing, spin tunneling, and kinetic exchange coupling in coupled quantum dot systems.

The paper is organized as follows. In Sec. II we introduce the model, the numerical technique, and materials and system parameters. In Sec. III we review the benchmark case of single dots with spin-orbit coupling and magnetic field. Coupled double dots are studied in Sec. IV, separately in zero and finite magnetic fields. We conclude with the discussion of our results in Sec. V.

\section{MODEL}

We consider a two-dimensional electron system confined in a [001] plane of a zinc-blende semiconductor heterostructure, with additional confinement into lateral dots given by appropriately shaped top gates. A magnetic field $\mathbf{B}$ is applied perpendicular to the plane. In the effective mass approximation the single-electron Hamiltonian of such a system, taking into account spin-orbit coupling, can be decomposed into several terms:

$$
H=T+V_{C}+H_{Z}+H_{B R}+H_{D}+H_{D 3} .
$$

Here $T=\hbar^{2} \mathbf{K}^{2} / 2 m$ is the kinetic energy with the effective electron mass $m$ and kinetic momentum $\hbar \mathbf{K}=\hbar \mathbf{k}+e \mathbf{A}$ $=-i \hbar \nabla+e \mathbf{A} ; e$ is the proton charge and $\mathbf{A}=B(-y / 2, x / 2,0)$ is the vector potential of $\mathbf{B}=(0,0, B)$. Vectors of position and $\mathbf{r}$ and momentum $\hbar \mathbf{k}$ are two dimensional. Operators of angular momentum with mechanical and canonical momenta are denoted as $\mathbf{L}=\mathbf{r} \times(\hbar \mathbf{K})$ and $\mathbf{l}=\mathbf{r} \times(\hbar \mathbf{k})$. The quantum dot geometry is described by the confining potential $V_{C}(\mathbf{r})$. Single dots will be described here by a parabolic potential $V_{C}=(1 / 2) m \omega_{0}^{2} r^{2}$, characterized by confinement energy $E_{0}$ $=\hbar \omega_{0} / 2$ and confinement length $l_{0}=\left(\hbar / m \omega_{0}\right)^{1 / 2}$, setting the energy and length scales, respectively. Coupled double dots will be described by two displaced (along $\mathbf{x}$ ) parabolas:

$$
V_{C}^{d d}=\frac{1}{2} m \omega_{0}^{2}\left[\left(|x|-l_{0} d\right)^{2}+y^{2}\right]
$$

the distance between the minima is $2 d$ measured in the units of $l_{0}$. The Zeeman energy is given by $H_{Z}=\left(g^{*} / 2\right) \mu_{B} \sigma_{z} B$, where $g^{*}$ is the conduction band $g$ factor, $\mu_{B}$ is the Bohr magneton, and $\sigma_{z}$ is the Pauli matrix. In order to relate the magnetic moment of electrons to their orbital momentum, we will use dimensionless parameter $\alpha_{Z}=g^{*} \mathrm{~m} / 2 m_{e}$, where $m_{e}$ is the free electron mass.

Spin-orbit coupling gives additional terms in confined systems. ${ }^{7}$ The Bychkov-Rashba Hamiltonian, 5,6

$$
H_{B R}=\widetilde{\alpha}_{B R}\left(\sigma_{x} K_{y}-\sigma_{y} K_{x}\right),
$$

appears if the confinement is not symmetric in the growth direction (here $\mathbf{z}$ ). The strength $\widetilde{\alpha}_{B R}$ of the interaction can be tuned by modulating the asymmetry by top gates. Due to the lack of spatial inversion symmetry in zinc-blende semiconductors, the spin-orbit interaction of conduction electrons takes the form of the Dresselhaus Hamiltonian ${ }^{4}$ which, when quantized in the growth direction $\mathbf{z}$ of our heterostructure, gives two terms, one linear and one cubic in momentum: ${ }^{45}$

$$
\begin{gathered}
H_{D}=\gamma_{c}\left\langle K_{z}^{2}\right\rangle\left(-\sigma_{x} K_{x}+\sigma_{y} K_{y}\right), \\
H_{D 3}=\left(\gamma_{c} / 2\right)\left(\sigma_{x} K_{x} K_{y}^{2}-\sigma_{y} K_{y} K_{x}^{2}\right)+\text { H.c. },
\end{gathered}
$$

where $\gamma_{c}$ is a material parameter. The angular brackets in $H_{D}$ denote quantum averaging in the $\mathbf{z}$ direction - the magnitude of $H_{D}$ depends on the confinement strength. We will denote the sum of the two linear spin-orbit terms by $H_{\text {lin }}=H_{D}$ $+\mathrm{H}_{B R}$. The complete spin-orbit coupling is then $H_{S O}=H_{\text {lin }}$ $+H_{D 3}$. We find it useful to introduce dimensionless strengths of the individual terms of the spin-orbit interaction by relating them to the confinement energy of a single dot $E_{0}$. We denote $\alpha_{B R}=\tilde{\alpha}_{B R} / E_{0} l_{0}$ and $\alpha_{D}=\gamma_{c}\left\langle k_{z}^{2}\right\rangle / E_{0} l_{0}$ for linear terms, and $\alpha_{D 3}=\gamma_{c} / 2 E_{0} l_{0}^{3}$ for the cubic Dresselhaus term.

In our numerical examples we use $E_{0}=\hbar \omega_{0} / 2$ $=1.43 \mathrm{meV}$ for the confinement energy, which corresponds to the confinement length of $l_{0}=20 \mathrm{~nm}$. We further use bulk GaAs materials parameters: $m=0.067 m_{c}, g^{*}=-0.44$, and $\gamma_{c}$ $=27.5 \mathrm{eV} \AA^{3}$. For $\left\langle k_{z}^{2}\right\rangle$ we choose $5.3 \times 10^{-4} \AA^{2}$, which corresponds to $\gamma_{c}\left\langle k_{z}^{2}\right\rangle=14.6 \mathrm{meV} \AA$. This value of $\left\langle k_{z}^{2}\right\rangle$ corresponds to the ground state of a $6 \mathrm{~nm}$ thick triangular potential well. ${ }^{29}$ For $\widetilde{\alpha}_{B R}$ we choose a generic value of $4.4 \mathrm{meV} \AA$, which is in line of experimental observations. ${ }^{46,47}$ The dimensionless parameter of the Zeeman splitting then is $\alpha_{Z}$ $=-0.015$, while the relative strengths of the spin-orbit interactions are $\alpha_{B R} \approx 0.015, \alpha_{D} \approx 0.05$, and $\alpha_{D 3} \approx 0.001$. Except 
for anticrossings, the spin-orbit interaction is a small perturbation to the electronic structure; it is, however, essential for investigating spin structure.

Our analytical calculations will often refer to the Fock-Darwin $^{48,49}$ spectrum, which is the spectrum of Hamiltonian (1) for a single dot with $H_{S O}=0$. The corresponding wave functions $\Psi$ (expressed in polar coordinates $r$ and $\phi$ ), and energies $\epsilon$ are

$$
\begin{gathered}
\Psi_{n, l, \sigma}(r, \phi)=C \rho^{|l|} e^{-\rho^{2} / 2} L_{n}^{|l|}\left(\rho^{2}\right) e^{i l \phi} \xi(\sigma), \\
\epsilon_{n, l, \sigma}=2 E_{0} \frac{l_{0}^{2}}{l_{B}^{2}}(2 n+|l|+1)+B \frac{\hbar e}{2 m}\left(l+\alpha_{Z} \sigma\right),
\end{gathered}
$$

where $\rho=r / l_{B}$ is the radius in the units of the effective confinement length $l_{B}$, defined by $l_{B}^{2}=l_{0}^{2} / \sqrt{ }\left(1+B^{2} e^{2} l_{0}^{4} / 4 \hbar^{2}\right) ; n$ and $l$ are the principal and orbital quantum numbers, respectively, $C$ is the state dependent normalization constant, and $L_{n}^{|l|}$ are associated Laguerre polynomials. Spinors $\xi(\sigma)$ describe the spin $\sigma$ state of the electrons. Since the parabolic dot has rotational symmetry in the plane, the states have well defined orbital momentum $l$ and spin $\sigma$ in the $\mathbf{z}$ direction. We also introduce a useful dimensionless measure $\theta$ of the strength of the magnetic field induced confinement compared to the potential confinement: $\theta=B e l_{B}^{2} / 2 \hbar, 0<\theta<1$. The parameter $\theta$ gives the number of magnetic flux quanta through a circle with radius $l_{B}$. For large magnetic fields $\theta \approx 1-\left(2 \hbar / B e l_{0}^{2}\right)^{2} / 2$. The confining length can be expressed as $l_{B}=l_{0}\left(1-\theta^{2}\right)^{1 / 4}$.

As it is not possible to solve for the spectrum of Hamiltonian (1) analytically, we treat it numerically with the finite differences method using Dirichlet boundary conditions (vanishing of the wave function at boundaries). The magnetic field is included via the Peierls phase: if $H\left(\mathbf{r}_{i}, \mathbf{r}_{j}\right)$ is the discretized Hamiltonian connecting grid points $\mathbf{r}_{i}$ and $\mathbf{r}_{j}$ at $B=0$, the effects of the field are obtained by adding a gauge phase: $H\left(\mathbf{r}_{i}, \mathbf{r}_{j}\right) \exp \left[i(e / \hbar) \int_{\mathbf{r}_{i}}^{\mathbf{r}_{j}} \mathbf{A}\right.$.dll $]$. In our simulations we typically use $50 \times 50$ grid points. The resulting matrix eigenvalue problem is solved by the Lanczos diagonalization. The achieved accuracy is about $10^{-5}$.

\section{SINGLE DOTS}

As a starting point we review the effects of spin-orbit coupling in single dots. We are interested in the changes to the spectrum and, in particular, to the magnetic moment of the lowest states, that is, to the effective $g$ factor. The calculated spectrum of a single dot is shown in Fig. 1. There are three ways in which spin-orbit coupling influences the spectrum: (i) First, the levels are shifted, in proportion to $\alpha^{2}$ (by $\alpha$ here we mean any of $\alpha_{B R}, \alpha_{D}$, or $\alpha_{D 3}$ ). (ii) Second, the degeneracy at $B=0$ is lifted, again in proportion to $\alpha^{2}(1 \mathrm{~b})$. (iii) Finally, at some magnetic field the level crossing of the Fock-Darwin levels is lifted by spin-orbit coupling. The resulting level repulsion is linear in $\alpha(1 \mathrm{c})$. The states here are the spin hot spots, that is states in which both Pauli spin up and down species contribute significantly. ${ }^{19,32,39}$

The above picture can be understood from general symmetry considerations within the framework of perturbation
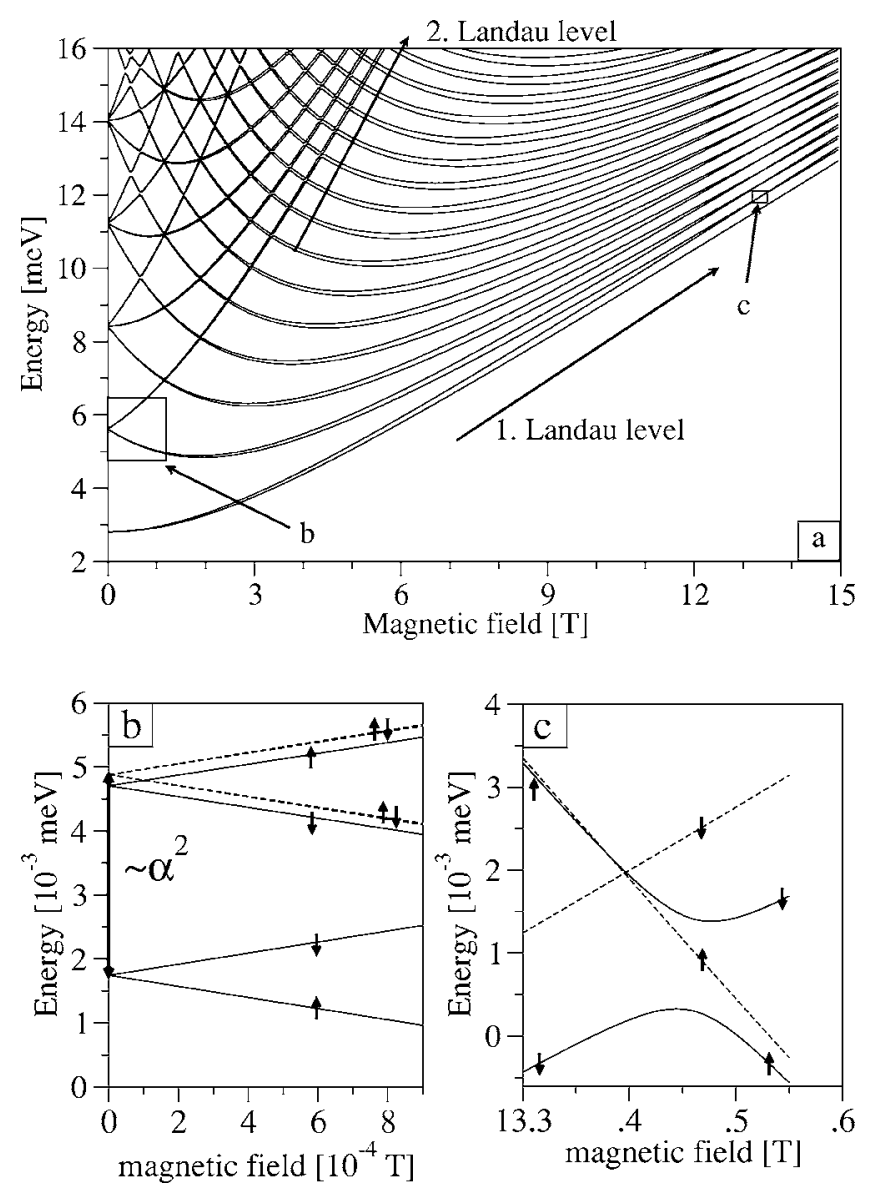

FIG. 1. Energy spectrum of a single dot in magnetic field. (a) The Fock-Darwin spectrum, Eq. (7). (b) Lowest orbital excited levels $(n=0,|l|=1)$ without (dashed) and with (solid) spin-orbit coupling. Arrows indicate the spin states. For clarity the energy's origin here is shifted relative to case (a). Both the shift in energy levels as well as the splitting at $B=0$ grow as $\alpha^{2}$. (c) Anticrossing at the critical magnetic field (here about $13 \mathrm{~T}$ ). For clarity, a linear trend was subtracted from the data.

theory. All spin-orbit terms commute, at $B=0$, with the time inversion operator $T=i \sigma_{y} \hat{C}$, where $\hat{C}$ is the operator of complex conjugation. Therefore Kramer's degeneracy is preserved so that the states are always doubly degenerate. The linear terms can be transformed into each other by a unitary transformation $\left(\sigma_{x}+\sigma_{y}\right) / \sqrt{2}$ (spin rotation around [110] by $\pi)$, which commutes with $H_{0}$. Therefore the effects on the energy spectrum induced individually by the linear Dresselhaus and the Bychkov-Rashba terms are identical at $B=0$. At finite magnetic fields the two interactions give qualitatively different effects in the spectrum, especially for spin hot spots, as discussed below.

For any $B$ the following commutation relations hold for the linear terms:

$$
\left[H_{B R}, l_{z}+s_{z}\right]=0, \quad\left[H_{D}, l_{z}-s_{z}\right]=0 .
$$

This means that $H_{B R}$ commutes with the angular momentum, while $H_{D}$ does not. This will influence the interference between the two terms in the energy spectrum. We can use the 
Fock-Darwin states as a basis for perturbation theory. Up to the second order the energy of state $|i\rangle=\Psi_{n, l, \sigma}$ is

$$
E_{i}=\epsilon_{i}+\left\langle i\left|H_{S O}\right| i\right\rangle+\sum_{j \neq i} \frac{\left\langle i\left|H_{S O}\right| j\right\rangle\left\langle j\left|H_{S O}\right| i\right\rangle}{\epsilon_{i}-\epsilon_{j}} .
$$

The first order correction is zero for all spin-orbit terms since $H_{S O}$ contain only odd powers of $\mathbf{K}$ whose expectation values in the Fock-Darwin states vanish. If the perturbation expansion is appropriate, the spin-orbit interactions have a second order (in $\alpha$ ) effect on energy.

Both linear spin-orbit terms couple states with orbital momenta $l$ differing by 1 . It then follows from the commutation relations (8) that $H_{B R}$ preserves the total angular momentum $l+s$, while $H_{D}$ preserves the quantity $l-s$. As a result, there is no correction to the energy from the interference terms between $H_{B R}$ and $H_{D}$ in Eq. (9): $\left\langle i\left|H_{B R}\right| j\right\rangle\left\langle j\left|H_{D}\right| i\right\rangle=0$. As for the cubic Dresselhaus term, only the following orbital states are coupled: $(l, \uparrow) \rightarrow\{(l+3, \downarrow),(l-1, \downarrow)\} \quad$ and $(l, \downarrow) \rightarrow\{(l-3, \uparrow),(l+1, \uparrow)\}$. Due to these selection rules there are no interference terms $\sim H_{D 3} H_{B R}$, but terms $\sim H_{D 3} H_{D}$ will contribute to energy perturbation. The Bychkov-Rashba and Dresselhaus Hamiltonians act independently on the Fock-Darwin spectrum (up to the second order).

To gain more insight into the perturbed structure of the spectrum at $B=0$, we rewrite Eq. (9) using an auxiliary antiHermitian operator $H_{S O}^{o p}$ defined by the commutation relation $\left[H_{0}, H_{S O}^{o p}\right]=H_{S O}$. If such an operator exists, the second order correction in (9) is then

$$
\begin{aligned}
\sum_{j \notin \mathcal{N}} \frac{\left\langle i\left|H_{S O}\right| j\right\rangle\left\langle j\left|H_{S O}\right| i\right\rangle}{\epsilon_{i}-\epsilon_{j}}= & \left\langle i\left|\frac{1}{2}\left[H_{S O}^{o p}, H_{S O}\right]\right| i\right\rangle \\
& +\operatorname{Re}\left(\left\langle i\left|H_{S O} P_{\mathcal{N}} H_{S O}^{o p}\right| i\right\rangle\right),
\end{aligned}
$$

where $P_{\mathcal{N}}$ is the projector on the subspace $\mathcal{N}$ of the states excluded from the summation. In our case here it is just one state, $\mathcal{N}=\{|i\rangle\}$. The last term in Eq. (10) then vanishes. The auxiliary operator for $H_{D 3}$ is not known and if found, it must depend on the confining potential. Operators for the linear terms are ${ }^{50}$

$$
\begin{aligned}
& H_{D}^{o p}=-i\left(\alpha_{D} / 2 l_{0}\right)\left(x \sigma_{x}-y \sigma_{y}\right), \\
& H_{B R}^{o p}=i\left(\alpha_{B R} / 2 l_{0}\right)\left(y \sigma_{x}-x \sigma_{y}\right) .
\end{aligned}
$$

The corresponding commutators are (in the zero magnetic field $\mathbf{K}=\mathbf{k}, L_{z}=l_{z}, \theta=0$; the last expression will be useful later)

$$
\begin{gathered}
{\left[H_{D}^{o p}, H_{D}\right]=-E_{0} \alpha_{D}^{2}\left(1-\sigma_{z} L_{z}\right),} \\
{\left[H_{B R}^{o p}, H_{B R}\right]=-E_{0} \alpha_{B R}^{2}\left(1+\sigma_{z} L_{z}\right),} \\
{\left[H_{D}^{o p}, H_{D 3}\right]=E_{0} l_{0}^{2} \alpha_{D} \alpha_{D 3}\left(K_{x}^{2}+K_{y}^{2}-2 \sigma_{z}\left[x K_{y} K_{x}^{2}-y K_{x} K_{y}^{2}\right.\right.} \\
\left.\left.-2 i \theta\left(x K_{x}+y K_{y}\right)\right]\right) .
\end{gathered}
$$

Because $\left[H_{D}^{o p}, H_{B R}\right]+\left[H_{B R}^{o p}, H_{D}\right]=0$, the corrections to the second order perturbation add independently for $H_{B R}$ and $H_{D}$ (as also noted above from the selection rules), we can intro- duce $H_{\operatorname{lin}}^{o p}=H_{D}^{o p}+H_{B R}^{o p}$. An alternative route to Eq. (10) is to transform the Hamiltonian with ${ }^{50} U=\exp \left(-H_{S O}^{o p}\right)$ to $\widetilde{H}$ $=H_{0}-(1 / 2)\left[H_{S O}, H_{S O}^{o p}\right]$ in the second order of $\alpha$. The final result can be also obtained in a straightforward way by using the Thomas-Reiche-Kuhn sum rule in the second order of the perturbation theory with the original spin-orbit terms. The resulting effective Hamiltonian is (terms depending on $\alpha_{D 3}$ are omitted here)

$$
\widetilde{H}=H_{0}-E_{0}\left(\alpha_{D}^{2}+\alpha_{B R}^{2}\right) / 2+E_{0} \sigma_{z} L_{z}\left(\alpha_{D}^{2}-\alpha_{B R}^{2}\right) / 2 .
$$

This Hamiltonian, in which the spin-orbit coupling appears in its standard form, neatly explains point (ii) about the lifting of the degeneracy at $B=0$. The levels in Fig. 1(b), for example, are four folddegenerate $(|l|=1,|\sigma|=1)$ without spin-orbit coupling. Turning on, say, $H_{D}$, will split the levels into two groups: energy of the states with $l \sigma>0$ would not change in the second order, while the states with $l \sigma<0$ will go down in energy by $E_{0} \alpha_{D}^{2}$, as seen in Fig. 1(b).

\section{A. Spin hot spots}

Spin hot spots are states formed by two or more states whose energies in the absence of spin-orbit coupling are degenerate or close to being degenerate, while turning on the coupling removes the degeneracy. ${ }^{39}$ Such states are of great importance for spin relaxation, which is strongly enhanced by their presence. ${ }^{19,51}$ The reason is that the degeneracy lifting mixes spin up and spin down states and so transitions between states of opposite magnetic moment will involve spin flips with a much more enhanced probability compared to normal states.

Figure 1(c) shows an interesting situation where two degenerate levels are lifted by spin-orbit coupling. ${ }^{19,32}$ The lifting is of the first order in $\alpha$, unlike the lifting of degeneracy at $B=0$ in which case the degenerate states are not directly coupled by $H_{S O}$. In a finite magnetic field, at a certain value $B_{\text {acr }}$, the states of opposite spins and orbital momenta differing by 1 cross each other, as follows from the Eq. (7). The crossing field is $B_{\text {acr }} \approx\left|\alpha_{Z}\right|^{-1 / 2} \hbar /\left(e l_{0}^{2}\right)$, which is about $13.4 \mathrm{~T}$ for our parameters (making the confinement length larger the magnitude of the field would decrease). Spin-orbit interaction couples the two states thereby lifting the degeneracy. For GaAs, where $g^{*}<0$, only the Bychkov-Rashba term couples the two states. The Dresselhaus terms are not effective $\left(H_{D 3}\right.$ would introduce such a splitting at $3 B_{\text {acr }}$ ). The energy splitting due to $H_{B R}$ is

$$
\Delta=c \sqrt{8} E_{0} \alpha_{B R}\left|\alpha_{Z}\right|^{5 / 4},
$$

where $c$, which is a number of order 1 , depends on the quantum numbers of the two states. Since spin hot spots at $B_{\text {acr }}$ are due to $H_{B R}$ only, the splittings could help to sort out the Bychkov-Rashba versus Dresselhaus contributions. Figure 1(c) shows the calculated level repulsion for states $n=0, l$ $=0, \sigma=\downarrow$ and $n=0, l=-1, \sigma=\uparrow$. The magnitude of $\Delta$, though being linear in $\alpha_{B R}$, is on the order of $10^{-3} \mathrm{meV}$ and thus comparable to the energy scales associated with quadratic spin-orbit perturbations. 


\section{B. Effective $g$ factor}

When probing spin states in quantum dots with magnetic field, important information comes from the measured Zeeman splitting. We will focus here on the two lowest spin states and calculate the effective $g$ factor as $g=\left(E_{0,0, \downarrow}\right.$ $\left.-E_{0,0, \uparrow}\right) /\left(\mu_{B} B\right)$. If $H_{S O}=0$, then in our model the effective $g$ factor is equal to the conduction band value $g^{*}$. In fact the $g$ factor is modified by also other confinement effects, ${ }^{52}$ but here we consider only spin-orbit interactions. The actual value in the presence of spin-orbit coupling is important for understanding single spin precession in magnetic field, which seems necessary to perform single-qubit operations in quantum dots. We have obtained the following contributions to the $g$ factor from nondegenerate (that is, excluding spin hot spots) second-order perturbation theory [Eq. (9)] (for linear spin-orbit terms these are derived also in Refs. 29 and 53):

$$
\begin{aligned}
\delta g_{D-D}= & -\frac{\alpha_{D}^{2}}{2 m / m_{e}} \frac{\sqrt{1-\theta^{2}}\left[1-\theta^{2}-2\left(1+\theta^{2}\right) \alpha_{Z}\right]}{1-\theta^{2}\left(1+4 \alpha_{Z}+4 \alpha_{Z}^{2}\right)}, \\
\delta g_{B R-B R}= & \frac{\alpha_{B R}^{2}}{2 m / m_{e}} \frac{\sqrt{1-\theta^{2}}\left[1-\theta^{2}+2\left(1+\theta^{2}\right) \alpha_{Z}\right]}{1-\theta^{2}\left(1-4 \alpha_{Z}+4 \alpha_{Z}^{2}\right)}, \\
\delta g_{D-D 3}= & \frac{\alpha_{D} \alpha_{D 3}}{m / m_{e}} \frac{\left(1+\theta^{2}\right)\left[1-\theta^{2}-2\left(1+\theta^{2}\right) \alpha_{Z}\right]}{1-\theta^{2}\left(1+4 \alpha_{Z}+4 \alpha_{Z}^{2}\right)}, \\
\delta g_{D 3-D 3}= & \frac{\alpha_{D 3}^{2}}{8 m / m_{e} \theta \sqrt{1-\theta^{2}}}\left(\frac{2(1-\theta)^{2}\left(1+\theta^{2}\right)^{2}}{1-\theta\left(1+2 \alpha_{Z}\right)}\right. \\
& +\frac{(1-\theta)^{4}(1+\theta)^{2}}{3-\theta\left(1+2 \alpha_{Z}\right)}+\frac{-3(1-\theta)^{6}}{3-\theta\left(3-2 \alpha_{Z}\right)} \\
& +\frac{3(1+\theta)^{6}}{3+\theta\left(3-2 \alpha_{Z}\right)}-\frac{2(1+\theta)^{2}\left(1+\theta^{2}\right)^{2}}{1+\theta\left(1+2 \alpha_{Z}\right)} \\
& \left.-\frac{(1-\theta)^{2}(1+\theta)^{4}}{3+\theta\left(1+2 \alpha_{Z}\right)}\right) .
\end{aligned}
$$

Here $\delta g_{A-B}$ stands for a correction that is proportional to $\alpha_{A} \alpha_{B}$.

The functions (18) are plotted in Fig. 2. We can understand the limits of $\delta g$ at $B \rightarrow \infty(\theta \rightarrow 1)$ if we notice that in the natural length unit $l_{B}$ the momentum $K_{x}=-i \partial_{x}-y B e / 2 \hbar$ $=l_{B}^{-1}\left[-i \partial_{x / l_{B}}-\theta\left(y / l_{B}\right)\right]$. In the limit $B \rightarrow \infty$ the matrix elements of $H_{D}$, which is linear in $K$, scale as $l_{B}^{-1}$, while the FockDarwin energies scale as $l_{B}^{-2}$. The second order $D-D$ correction to $E_{0,0, \downarrow}-E_{0,0, \uparrow}$ is thus independent of $l_{B}$; it converges to $-E_{0} \alpha_{D}^{2} /\left(1+\alpha_{Z}\right)$. The $B R-B R$ correction is analogous, with the limit $E_{0} \alpha_{B R}^{2} /\left(1-\alpha_{Z}\right)$. To get the $g$ factor we divide the energy differences by $\mu_{B} B$ and get $\delta g_{D-D}(\theta \rightarrow 1) \propto B^{-1}$; similarly for $H_{B R}$. Since $H_{D 3}$ scales as $l_{B}^{-3}$ one gets $\delta g_{D-D 3}(\theta \rightarrow 1)$ $\rightarrow 2 \alpha_{D} \alpha_{D 3} m /\left(1+\alpha_{Z}\right) m_{e}$ and $\delta g_{D 3-D 3}(\theta \rightarrow 1) \propto B$. It seems that by increasing $B$ there inevitably comes a point where the influence of $H_{D 3}$ on the $g$ factor dominates. But at $B=B_{\text {acr }}$ there is an anticrossing of the states $(0,0, \downarrow)$ and $(0,-1, \uparrow)$ so for larger $B$ the $g$ factor does not describe the energy difference between the two lowest states, but rather between

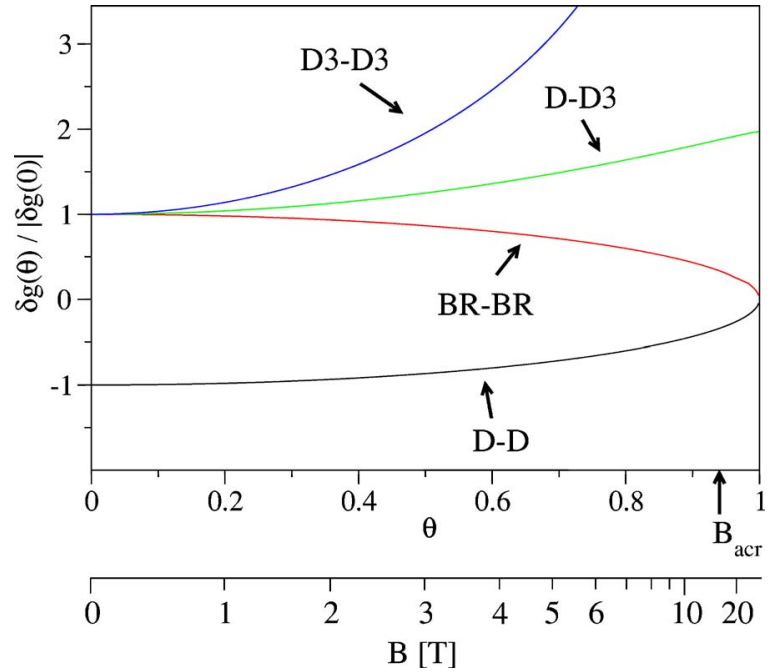

FIG. 2. (Color online) Calculated corrections to the effective $g$ factor by spin-orbit interactions. Formulas (18) scaled by the values at $B=0$ (and thus independent on $\alpha_{S O}$ ) are plotted. The actual numerical values of $\delta g$ at $B=0$ are $\delta g_{D-D}(0)=1.0 \times 10^{-2}, \delta g_{B R-B R}(0)$ $=8.6 \times 10^{-4}, \delta g_{D-D 3}(0)=9.4 \times 10^{-4}$, and $\delta g_{D 3-D 3}(0)=2.5 \times 10^{-5}$. At the anticrossing $\delta g_{D-D}\left(B_{\text {acr }}\right)=2.4 \times 10^{-3}, \delta g_{B R-B R}\left(B_{\text {acr }}\right)=1.0 \times 10^{-4}$, $\delta g_{D-D 3}\left(B_{\text {acr }}\right)=1.8 \times 10^{-3}$, and $\delta g_{D 3-D 3}\left(B_{\text {acr }}\right)=3.4 \times 10^{-4}$.

the second excited state and the ground state. The value of $B$ where $\delta g_{D 3-D 3}=\delta g_{D-D}$ is given by $B \approx\left(\hbar / e l_{0}^{2}\right) \alpha_{D} / \alpha_{D 3} \sqrt{2}$. For GaAs parameters it is $\approx 25 \mathrm{~T}$.

\section{DOUBLE DOTS}

A double dot structure comprises two single dots close enough for their mutual interaction to play an important role. Here we consider symmetric dots modeled by $V_{C}^{d d}$ of Eq. (2). Such a potential has an advantage that in the limits of small $d \rightarrow 0$ and large $d \rightarrow \infty$, the solutions converge to the single dot solutions centered at $x=0$ and $\pm l_{0} d$, respectively. We denote the displaced Fock-Darwin states as $\Psi_{n, l, \sigma}^{ \pm d}(x, y)$ $\equiv \Psi_{n, l, \sigma}\left(x \pm l_{0} d, y\right)$.

The symmetries of the double dot Hamiltonian with spinorbit couplings are listed in Table I. The time symmetry is

TABLE I. Symmetries of the double dot Hamiltonian for different spin-orbit terms present at $B=0$ and $B>0$. Here $I_{x}\left(I_{y}\right)$ means reflection $x \rightarrow-x(y \rightarrow-y), I=I_{x} I_{y}$, and $R_{z}=\exp \left(-i \phi \sigma_{z} / 2\right)$ is the rotation of a spinor by angle $\phi$ around $z$ axis; $R_{\mathbf{n}}$ is a spinor rotation around an arbitrary axis $\mathbf{n}$ and $T$ is the time reversal symmetry. The identity operation is not listed.

\begin{tabular}{ccc}
\hline \hline Magnetic field & SO terms & Symmetries of $H$ \\
\hline \multirow{2}{*}{$B=0$} & none & $I_{x}, I_{y}, I, T, R_{\mathbf{n}}$ \\
& $B R$ & $-i \sigma_{x} I_{x},-i \sigma_{y} I_{y},-i \sigma_{z} I, T$ \\
& $D, D 3$ & $-i \sigma_{y} I_{x},-i \sigma_{x} I_{y},-i \sigma_{z} I, T$ \\
& all & $-i \sigma_{z} I, T$ \\
$B>0$ & none & $-i \sigma_{z} I, R_{z}$ \\
& any & $-i \sigma_{z} I$ \\
\hline \hline
\end{tabular}


TABLE II. Notation and transformation properties of $\mathrm{C}_{2 v}$ representations. $L$ and $D$ are the coefficients of the dependence of $g_{i}^{n, l, \sigma}$ on the single dot functions (see text).

\begin{tabular}{ccccccc}
\hline \hline & & \multicolumn{4}{c}{ Numbers for $g_{i}^{n, l, \sigma}$} \\
\cline { 3 - 6 } Representation & $\begin{array}{c}\text { Under } I_{x}, I_{y} \\
\text { transforms }\end{array}$ & \multicolumn{2}{c}{$l$ even } & \multicolumn{2}{c}{$l$ odd } \\
\hline$\Gamma_{1}$ & as & $\mathrm{L}$ & $\mathrm{D}$ & $\mathrm{L}$ & $\mathrm{D}$ \\
$\Gamma_{2}$ & 1 & 1 & 1 & -1 & -1 \\
$\Gamma_{3}$ & $x$ & -1 & -1 & 1 & 1 \\
$\Gamma_{4}$ & $x y$ & -1 & 1 & 1 & -1 \\
\hline \hline
\end{tabular}

always present at $B=0$, giving Kramer's double degeneracy. The rotational space symmetry from the single dot case is lost; instead there are two discrete symmetries-reflections $I_{x}$ about $y$ and $I_{y}$ about $x$. In zero magnetic field and without spin-orbit terms, the Hamiltonian has both $I_{x}$ and $I_{y}$ symmetries. When only one of the spin-orbit terms is present (Rashba or Dresselhaus), we can still preserve symmetries $I_{x}$ and $I_{y}$ by properly defining the symmetry operators to act also on the spinors (forming the double group). The Bychkov-Rashba term, $H_{0}+H_{B R}$, is invariant to operations defined by the spatial invariance. This is not the case for $H_{D}$, since here the operators $-i \sigma_{y} I_{x}$ and $-i \sigma_{x} I_{y}$ do not describe a spatial reflection of both the orbital and spinor parts. The symmetry operations for $H_{B R}$ and $H_{D}$ are connected by the unitary transformation $\left(\sigma_{x}+\sigma_{y}\right) / \sqrt{2}$, which connects the two Hamiltonians themselves. Finally, if both spin-orbit terms are present, or at $B>0$, the only space symmetry left is $I=I_{x} I_{y}$.

In the following we consider separately the cases of zero and finite magnetic fields.

\section{A. Energy spectrum in zero magnetic field, without spin-orbit terms}

If no spin-orbit terms are present the group of our double dot Hamiltonian is $\mathrm{C}_{2 v} \otimes \mathrm{SU}(2)$. The $\mathrm{SU}(2)$ part accounts for the (double) spin degeneracy. The orbital parts of the eigenstates of the Hamiltonian therefore transform according to the irreducible representations of $\mathrm{C}_{2 v}$. The representations ${ }^{54}$ $\Gamma_{i}, i=1, \ldots, 4$, along with their transformation properties under the symmetries of $\mathrm{C}_{2 v}$, are listed in Table II. The symmetry properties will be used in discussing the perturbed spectrum.

We denote the exact eigenfunctions of the double dot Hamiltonian as $\Gamma_{i \sigma}^{a b}$ where $a(b)$ is the single dot level to which this eigenfunction converges as $d \rightarrow 0(\infty)$; $i$ labels the irreducible representation, $\sigma$ denotes spin. We have chosen the confining potential to be such that at $d \rightarrow 0(\infty)$ the solutions of the double dot $H_{0}$ converge to the (shifted) FockDarwin functions, if properly symmetrized according to the representations of $\mathrm{C}_{2 v}$. These symmetrized functions will be denoted as $g_{i}^{n, l, \sigma}$, where (up to normalization)

$$
g_{i}^{n, l, \sigma}=\left(\Psi_{n, l, \sigma}^{d}+D_{i} \Psi_{n, l, \sigma}^{-d}\right)+L_{i}\left(\Psi_{n,-l, \sigma}^{d}+D_{i} \Psi_{n,-l, \sigma}^{-d}\right) .
$$

The numbers $D_{i}\left(L_{i}\right)$ for different irreducible representations are in the Table II.

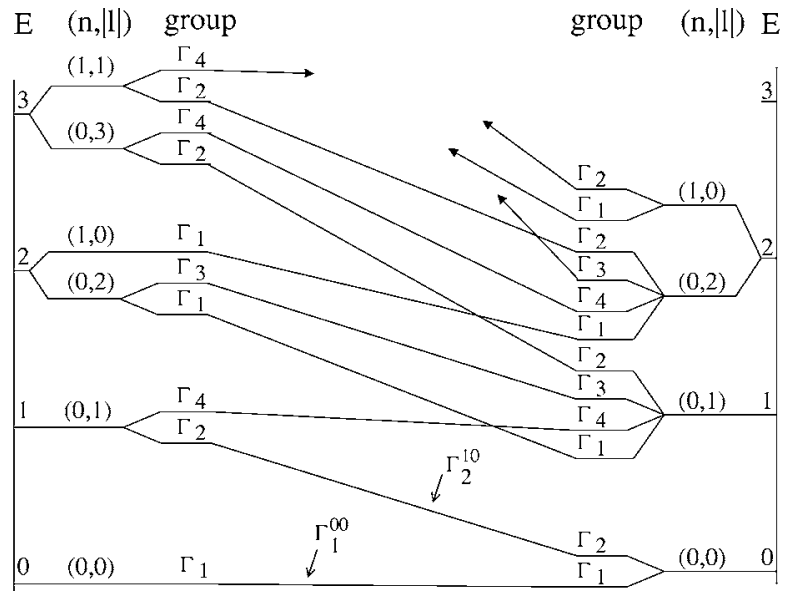

$d=0$

d

$\mathrm{d}=\infty$

FIG. 3. Single electron spectrum of a symmetric $\left(\mathrm{C}_{2 v}\right)$ lateral double dot structure as a function of the interdot separation, at $B$ $=0$, derived by applying group theoretical considerations. Single dot states at $d=0$ and $d=\infty$ are labeled by the principal $(n)$ and orbital $(l)$ quantum numbers, while the double dot states are labeled according to the four irreducible representations $\Gamma_{i}$ of $\mathrm{C}_{2 v}$. The lowest double dot states have explicitly excitation level of the $d$ $=0$ and $d=\infty$ states they connect. Every state is doubly (spin) degenerate, and the spin index is not given.

Generally, up to normalization, the exact solution can be written as a linear combination of any complete set of functions (we omit the spin index which is the same for all terms in the equation)

$$
\Gamma_{i}^{a b}=\sum_{n, l} \tilde{c}(n, l) g_{i}^{n, l}=g_{i}^{n_{0}, l_{0}}+\sum_{n, l} c(n, l) g_{i}^{n, l}
$$

The last equation indicates the fact, that for a function $\Gamma_{i}^{a b}$ in the limit $d \rightarrow 0(\infty)$, there will be a dominant $g$ function in the sum with the numbers $n_{0}, l_{0}$ given by the level $a(b)$ and the coefficients $c$ for the other functions will converge to zero. We term the approximation $c(n, l)=0$ as a linear combination of single dot orbitals (LCSDO).

Knowing the representations of the double dot Hamiltonian and the fact that Fock-Darwin functions form $\mathrm{SO}(2)$ representations (reflecting the symmetry of single dot $H_{0}$ ) we can decompose all single dot levels into the double dot representations and thus formally construct the energy spectrum of a double dot using the symmetry considerations only. Following the standard technique for constructing such correlation diagrams (connecting states of the same representation and avoiding crossing of lines of the same representation) we arrive at the spectrum shown in Fig. 3. The ground state transforms by the symmetry operations according to $\Gamma_{1}$ (identity), while the first excited state according to $\Gamma_{2}(x)$. This is expected for the symmetric and antisymmetric states formed by single dot ground states. The symmetry structure of the higher excited states is important to understand spinorbit coupling effects. Indeed, the spin-orbit terms couple two opposite spins according to certain selection rules. Since $H_{D}$, for example, transforms similarly to $x \oplus y$, it couples the 


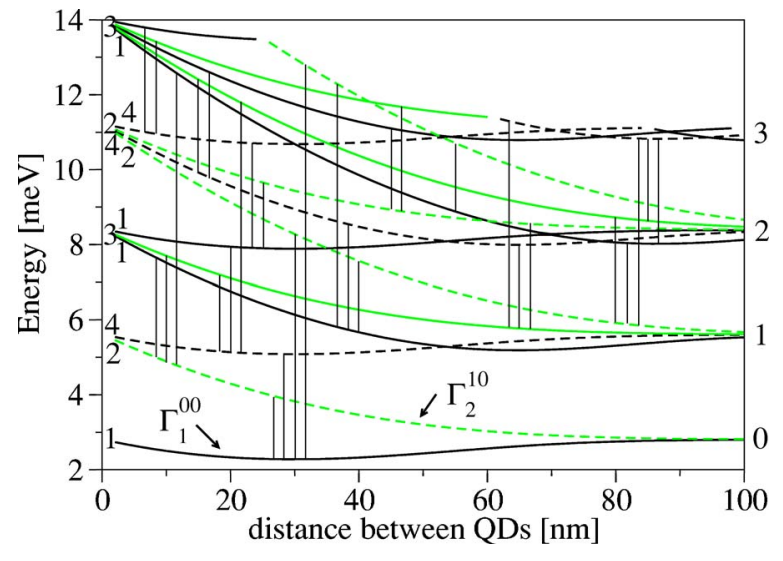

FIG. 4. (Color online) Calculated energy spectrum of a double quantum dot at $B=0$, as a function of interdot distance. Spindependent terms are not included in the Hamiltonian. Vertical bars indicate couplings due to spin-orbit interactions. Group theoretical symbols are shown with the lines on the left. Single dot levels are denoted by the highest orbital momentum $(0,1,2, \ldots)$ present in the degenerate set. This labeling is on the right. Every state is doubly degenerate, and the spin index is not given.

ground state $\Gamma_{1}$ with $\Gamma_{2}$ and $\Gamma_{4}$. In general, odd numbered representations can couple to even numbered representations. The same holds for $H_{B R}$ and $H_{D 3}$. If we include either $H_{B R}$ or $H_{D}$ into the Hamiltonian, and consider spinors as the basis for a representation, the states would transform according to $\Gamma_{5}$, the only irreducible representation of the double group of $\mathrm{C}_{2 v}$.

The calculated numerical spectrum for our model structure is shown in Fig. 4. There is a nice qualitative correspondence with Fig. 3. In Fig. 4 by vertical bars we denote coupling through $H_{D}$ or $H_{B R}\left(\left|\left\langle i\left|H_{D}\right| j\right\rangle\right|=\left|\left\langle i\left|H_{B R}\right| j\right\rangle\right|\right)$. The couplings follow the selection rule described above. Since there are several level crossings in the lowest part of the spectrum, a question arises if spin hot spots are formed in the presence of spin-orbit coupling. It turns out that there is no first-order level repulsion at the crossings due to the linear spin-orbit terms because the levels are not coupled by the linear terms, even though such couplings are allowed by symmetry. There are no spin hot spots due to the linear spin-orbit terms at zero magnetic field. For example $\Gamma_{4}^{11}$ and $\Gamma_{1}^{21}$ are not coupled by spin-orbit terms, and therefore their degeneracy (at $2 d l_{0} \approx 50 \mathrm{~nm}$ ) is not lifted by linear spin-orbit terms as we would expect from symmetry (actually, there is an anticrossing which is of the order $\alpha_{\text {lin }}^{3}$, instead of the expected $\alpha_{\text {lin }}$ ). The cubic Dresselhaus term gives here (and also in other crossings that conform with the selection rules) a linear anticrossing, as one expects. The absence of anticrossings from the linear spin-orbit terms will be explained in the next section.

Since our main goal here is to study the effects of spinorbit coupling on the tunneling between the two dots, we first look at the tunneling for $H_{S O}=0$. We use the LCSDO approximation for the wave function $\Gamma_{i}$ and compute energy as $E_{i}=\left\langle\Gamma_{i} \mid H \Gamma_{i}\right\rangle /\left\langle\Gamma_{i} \mid \Gamma_{i}\right\rangle$. We denote the energies of the two lowest orbital double dot states $\Gamma_{1 \sigma}^{00} \equiv \Gamma_{S}^{\sigma}, \Gamma_{2 \sigma}^{10} \equiv \Gamma_{A}^{\sigma}$ as $E_{S}^{(0)}, E_{A}^{(0)}$, where index zero indicates the absence of spin-orbit coupling. We obtain

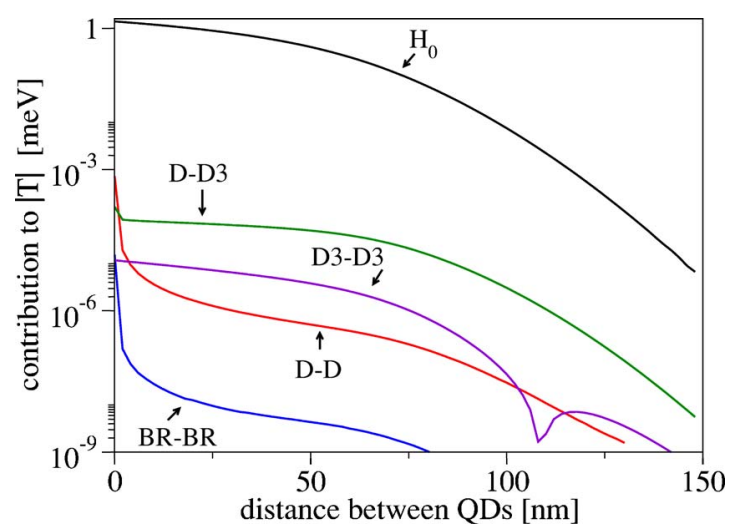

FIG. 5. (Color online) Calculated corrections to the tunneling energy $T$ from spin-orbit terms at $B=0$. The labels indicate which spin-orbit terms are involved. Only $D-D 3$ and $D 3-D 3$ are of second order. The remaining contributions are of fourth order.

$$
\begin{gathered}
E_{S}^{(0)}=2 E_{0} \frac{1+[1-2 d / \sqrt{\pi}] e^{-d^{2}}+d^{2} \operatorname{Erfc}(d)}{1+e^{-d^{2}}}, \\
E_{A}^{(0)}=2 E_{0} \frac{1-e^{-d^{2}}+d^{2} \operatorname{Erfc}(d)}{1-e^{-d^{2}}} .
\end{gathered}
$$

In the limit of large interdot separation the tunneling energy, $T=\left(E_{A}-E_{S}\right) / 2$, becomes,

$$
T^{(0)} \approx E_{0} \frac{2}{\sqrt{\pi}} d e^{-d^{2}} .
$$

It turns out that going beyond LCSDO does not improve the calculated $T^{(0)}$ significantly. The tunneling computed by full formulas, Eq. (21), does not differ from the numerically obtained value by more than $2 \%$ for any value of the interdot distance; the leading order becomes an excellent approximation for interdot distances larger than $50 \mathrm{~nm}$.

\section{B. Corrections to energy from spin-orbit coupling in zero magnetic field}

When we add $H_{S O}$ to $H_{0}$, the structure of the corrections to the energies of the two lowest states up to the second order in spin-orbit couplings can be expressed as

$$
E_{i}^{(2)}=-\mathcal{A}_{i}\left(\alpha_{D}^{2}+\alpha_{B R}^{2}\right)-\mathcal{B}_{i} \alpha_{D 3}^{2}+\mathcal{C}_{i} \alpha_{D} \alpha_{D 3},
$$

where $i$ is either $S$ or $A$. For the two lowest states the coefficients $\mathcal{A}, \mathcal{B}$, and $\mathcal{C}$ are positive for all values of the interdot distance and the differences $\mathcal{A}_{A}-\mathcal{A}_{S}, \ldots$ approach zero as $d \rightarrow \infty$. We will argue below that $\mathcal{A}_{S}=\mathcal{A}_{A}=\frac{1}{2}$ with the exception of a very small interdot distance (less than $1 \mathrm{~nm}$ ). There are thus no contributions from the linear spin-orbit couplings to $T$ in the second order. Only the cubic Dresselhaus term contributes, either by itself or in combination with the linear Dresselhaus term. Spin-dependent tunneling is greatly inhibited.

Numerical calculations of the corrections to $T$ from spinorbit couplings are shown in Fig. 5 The dominant correction 
is the mixed $D-D 3$ term, followed by the $D 3-D 3$ term. These are the only second order corrections. For GaAs, and our model geometry, these corrections are about 4 and 5 orders of magnitude lower than $T^{(0)}$. The linear spin-orbit corrections $(D-D$ and $B R-B R)$ are much smaller since they are of the fourth order. The dramatic enhancement of the corrections from linear spin-orbit terms close to $d=0$ is due to the transition from coupled to single dots. We will explore this region in more detail later.

We first show that a naive approach to calculate spin-orbit contributions to tunneling fails to explain the above results. We use the example of the linear Dresselhaus term. The simplest way to include this term is to begin with the two lowest orbital states (that are four states including spin), $g_{1}^{0,0, \sigma}$ and $g_{2}^{0,0, \sigma}$ and diagonalize the Hamiltonian in this basis. Because of the time reversal symmetry the resulting $4 \times 4$ matrix block diagonalizes into two equal $2 \times 2$ matrices with elements $H_{11}=E_{S}^{(0)}, \quad H_{22}=E_{A}^{(0)}, \quad$ and $H_{12}=\left\langle g_{1}^{0,0, \uparrow}\left|H_{D}\right| g_{2}^{0,0, \downarrow}\right\rangle$ $=-i E_{0} \alpha_{D} d e^{-d^{2}}$. Using the large $d$ limit for $T^{(0)}$, Eq. (22), we obtain the perturbed energies $E_{S(A)}=2 E_{0} \pm E_{0} \sqrt{4 / \pi+\alpha_{D}^{2}} d e^{-d^{2}}$ with the minus (plus) sign for $S(A)$. In the second order of $\alpha_{D}$ the symmetric and antisymmetric level energies have opposite contributions, giving $T \approx\left[\left(2 E_{0} / \sqrt{\pi}\right)\right.$ $\left.+\left(E_{0} \sqrt{\pi} / 4\right) \alpha_{D}^{2}\right] d \exp \left(-d^{2}\right)$, in contrast to the numerical results where there is no dependence on $\alpha_{D}^{2}$ in the second order. A larger basis, as well as a small basis comprising single dot states including spin-orbit coupling, still yield the $\alpha_{D}^{2}$ dependence.

From the previous example one can see that to get a correct (constant) spin-orbit contribution to the energy of a state, it is not enough to include a finite number of terms in the sum in Eq. (9). Instead we employ the operators $H^{o p}$ given in Eqs. (11) and (12). To get a contribution for a particular state, say $|i\rangle$, we apply the Löwdin perturbation theory. ${ }^{55}$ For this one has to identify states $|j\rangle$ which are degenerate with $|i\rangle$ with respect to the perturbation $H_{S O}$ and these have to be treated exactly. The rest of the states can be treated perturbatively. The condition for a degeneracy of two states can be taken as $\left|E_{i}^{(0)}-E_{j}^{(0)}\right| \lesssim \alpha_{\operatorname{lin}}\left(\alpha_{D 3}\right)$, when one considers linear (cubic) terms. The finite set of the degenerate states will be denoted by $\mathcal{N}$. The effective Hamiltonian $H^{\text {eff }}$ acting in $\mathcal{N}$ is

$$
\begin{aligned}
H_{i j}^{\mathrm{eff}}= & \left(H_{0}+H_{S O}\right)_{i j}+\frac{1}{2} \sum_{k \notin \mathcal{N}}\left(\frac{\left(H_{S O}\right)_{i k}\left(H_{S O}\right)_{k j}}{E_{i}^{(0)}-E_{k}^{(0)}}\right. \\
& \left.+\frac{\left(H_{S O}\right)_{i k}\left(H_{S O}\right)_{k j}}{E_{j}^{(0)}-E_{k}^{(0)}}\right) .
\end{aligned}
$$

For the example of the linear Dresselhaus term, we can now use Eqs. (10) and (13) to obtain

$$
H_{i j}^{\mathrm{eff}}=\left(H_{0}+H_{D}\right)_{i j}-\frac{1}{2} \alpha_{D}^{2} E_{0}\left(1-\sigma_{z} l_{z}\right)_{i j}+R_{i j},
$$

where

$$
R_{i j}=\frac{1}{2}\left\langle i\left|H_{D} P_{\mathcal{N}} H_{D}^{o p}-H_{D}^{o p} P_{\mathcal{N}} H_{D}\right| j\right\rangle .
$$

First we note that existence of the operator $H_{D}^{o p}$ means that the coupling through $H_{D}$ between any two states is always much smaller than the difference of the unperturbed energies of these two states, since $\left(H_{D}\right)_{i j}=\left(E_{i}^{(0)}-E_{j}^{(0)}\right)\left(H_{D}^{o p}\right)_{i j} \sim\left(E_{i}^{(0)}\right.$ $\left.-E_{j}^{(0)}\right) \alpha_{D}$. Then one can partially diagonalize the effective Hamiltonian to eliminate the off-diagonal $H_{D}$ terms. It turns out that this leads to a cancellation of the terms $H_{D}$ and $R$. The effective Hamiltonian is then

$$
H_{i j}^{\mathrm{eff}}=\left(H_{0}\right)_{i j}-\frac{1}{2} \alpha_{D}^{2} E_{0}\left(1-\sigma_{z} l_{z}\right)_{i j} .
$$

This completes the way to obtain Eq. (16) using Löwdin perturbation theory. There are no linear effects on the double dot energy spectrum from linear spin-orbit terms, which explains the absence of spin hot spots even though symmetry allows these hot spots to exist.

The spin-orbit interaction influences the energy only through the operator $l_{z}$, which is of the representation $\Gamma_{3}$, from where we get the selection rule-the allowed coupling is between functions of representations $\Gamma_{1}-\Gamma_{3}$ and $\Gamma_{2}-\Gamma_{4}$. Looking at Fig. 4, accidental degeneracies of states with such representations are not present in the lower part of the spectrum. The crossing of $\Gamma_{1}^{21}$ with $\Gamma_{4}^{11}$ considered in the discussion of Fig. 4 also does not follow the selection rule, hence why the anticrossing is of the third order. From the selection rules one can immediately see that also the expectation value of $l_{z}$ is zero in any state. This result is more general and holds also if the symmetry of the potential is lower (or none), since it follows from the fact that the Hamiltonian $H_{0}$ is real, so one can choose eigenfunctions to be real. Then the expectation value of any imaginary operator, such as $l_{z}$, must vanish. We conclude, that apart from degeneracies following from the single dot [that is limits $d \rightarrow 0(\infty)$ ] and possible accidental degeneracies respecting the selection rule, double dot states are nondegenerate and described by a $1 \times 1$ effective Hamiltonian

$$
H_{i i}^{\mathrm{eff}}=E_{i}^{(0)}-\frac{1}{2} E_{0} \alpha_{D}^{2}
$$

Particularly, the energies of the two lowest states are given by this equation, with an exception for the state $\Gamma_{A}$ in the region of small $d$ where it is coupled to $\Gamma_{4}^{11}$ through $l_{z}$ and we have to describe it here by a $2 \times 2$ effective Hamiltonian.

An illustration of the $l_{z}$ influence on the spectrum is in Fig. 6, where the linear Dresselhaus spin-orbit contribution to the energy for several states as a function of the interdot distance is shown. One can see at what interdot distances the $l_{z}$ operator causes the qualitative change between the double dot case (where the functions are characterized by a definite representation $\Gamma_{i}$ and the energy contribution from the spinorbit is a uniform shift) and the single dot case (where the functions are numbered according to the orbital momentum and the spin-orbit contribution to the energy depends on $\sigma_{z} l_{z}$ ). This happens when $E_{0} \alpha_{D}^{2}$ is comparable to the energy difference of the nearly degenerate states. If the criterion for the coupling between the dots is the constant contribution, $-\alpha_{D}^{2} E_{0} / 2$, to the energy, then the double dot region, as far as the spin-orbit coupling is concerned, is between 1 to $100 \mathrm{~nm}$, that is up to 5 times of the confinement length of $20 \mathrm{~nm}$. As an example, for the function $\Gamma_{4}^{11}$ the coupling in the effective Hamiltonian through $l_{z}$ to $\Gamma_{2}^{31}$ is equal to the 


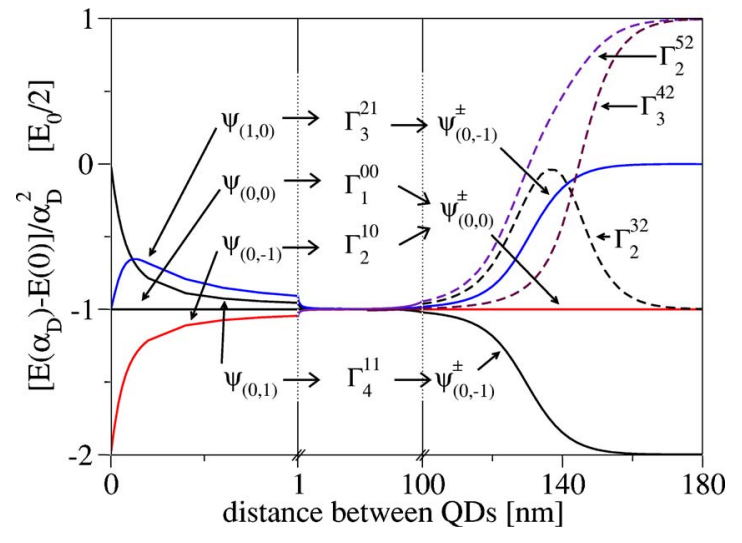

FIG. 6. (Color online) Calculated corrections to selected lowest energy levels due to $H_{D}$. All states have spin $\sigma=+1$. The graph demonstrates a transition between the symmetry group of the double dot $H_{0}$ (states $\Gamma$ ) and that of single dots (states $\Psi$ ). The transition is induced by $l_{z}$ which by symmetry couples states $\Gamma_{1} \leftrightarrow \Gamma_{3}$ and $\Gamma_{2} \leftrightarrow \Gamma_{4}$. Thus the anticrossing mechanism will induce transition $\Gamma_{1(3)} \leftrightarrow \Gamma_{1} \pm \Gamma_{3}$ and $\Gamma_{2(4)} \leftrightarrow \Gamma_{2} \pm \Gamma_{4}$. These linear combinations are equal to a single dot solution $\Psi_{n, l, \sigma}$ in the case $d \rightarrow 0$ and a combination $\Psi_{n, l, \sigma}^{ \pm} \equiv \Psi_{n, l, \sigma}^{d} \pm \Psi_{n, l, \sigma}^{-d}$ of functions with the same orbital momenta in the case $d \rightarrow \infty$.

unperturbed energy difference if $\alpha_{D}^{2} \sim d^{3} e^{-d^{2}}$, giving $d \approx 3$, corresponding to the interdot distance of $6 l_{0}$. Due to the exponential, this result is insensitive to $\alpha_{D}$.

The Bychkov-Rashba term can be treated analogously. The effective Hamiltonian is $H_{i j}^{\text {eff }}=\left[H_{0}-\left(\alpha_{B R}^{2} / 2\right) E_{0}(1\right.$ $\left.\left.+\sigma_{z} l_{z}\right)\right]_{i j}$. The absence of a linear influence on the energy was based on the existence of $H_{D}^{o p}$. Since we found a case where $H_{D 3}$ causes linear anticrossing (see discussion to Fig. 4), it follows that $H_{D 3}^{o p}$ cannot exist for our double dot potential. However, if one approximates $E_{i}-E_{k} \approx E_{j}-E_{k}$ in Eq. (24), one can use $H_{D}^{o p}$ to simplify the mixed $D-D 3$ correction. This, according to Fig. 5, is the dominant spin-orbit correction for the tunneling energy $T$. One gets an analogous expression as Eq. (25), where the needed commutator is stated in Eq. (15). Concluding, if we neglect the mixed D3-D3 term, we can write the spin-orbit contribution to the energy for the lowest orbital states to be $(i=S, A)$

$$
\delta E_{i}^{S O}=-E_{0}\left(\alpha_{D}^{2}+\alpha_{B R}^{2}\right) / 2+\alpha_{D} \alpha_{D 3} E_{0} l_{0}^{2}\left(\mathbf{k}^{2}\right)_{i i} .
$$

One note to the eigenfunctions: The matrix elements of the effective Hamiltonian are computed using the eigenfunctions of $H_{0}$. But the functions that correspond to the solutions are transformed by the same unitary transformation that leads from $H_{0}$ to $H^{\text {eff }}$. The sum rule can be used also here to express the influence of $H_{\text {lin }}$ on the eigenfunctions of $H_{0}$. If $H_{0} \Gamma_{i}=E_{i} \Gamma_{i}$, the eigenfunctions corresponding to the effective Hamiltonian, Eq. (25), are

$$
\widetilde{\Gamma}_{i}=\left(I+\sum_{j \notin \mathcal{N}} \frac{\left(H_{\mathrm{lin}}\right)_{j i}}{E_{i}-E_{j}}\right) \Gamma_{j}=\left[\mathbb{I}-\left(\mathbb{I}-P_{\mathcal{N}}\right) H_{\mathrm{lin}}^{o p}\right] \Gamma_{i} .
$$

Partial diagonalization of the effective Hamiltonian, to go from Eq. (25) to Eq. (27), means we finish the unitary transformation completely and get $\widetilde{\Gamma}_{i}^{\sigma}=\Gamma_{i}^{\sigma}-H_{\operatorname{lin}}^{o p} \Gamma_{i}^{\sigma}$ for the eigen-

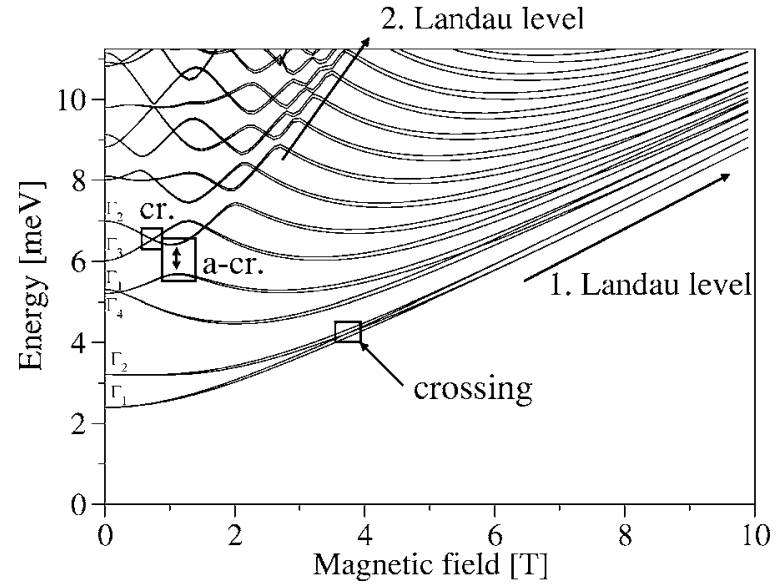

FIG. 7. Computed energy spectrum of the double dot Hamiltonian without $H_{S O}$, as a function of magnetic field. The quantum dot separation is $50 \mathrm{~nm}$ (single dot confinement length is $20 \mathrm{~nm}$ ). The energy levels are labeled according to the symmetry of the states at $B=0$. Two crossings (one between $\Gamma_{2}$ and $\Gamma_{3}$, the other between $\Gamma_{1}$ and $\Gamma_{2}$ ) and one anticrossing (between $\Gamma_{2}$ and $\Gamma_{4}$ ) are indicated. In the limit $B \rightarrow \infty$ the states merge to Landau levels.

functions corresponding to the effective Hamiltonian, Eq. (27).

\section{Finite magnetic field, no spin-orbit terms}

The presence of a magnetic field lowers the symmetry of the Hamiltonian without spin-orbit terms. The only nontrivial symmetry operator is the inversion I (see Table II). As a consequence the double dot states fall into two groups (representations of $C_{2}$ ): $\Gamma_{1}$ and $\Gamma_{3}$ become $\Gamma_{S}$ (symmetric under $I$ ) and $\Gamma_{2}$ and $\Gamma_{4}$ become $\Gamma_{A}$ (antisymmetric under $I$ ). Symmetrized functions $g_{i}^{n, l, \sigma}$ now are

$$
g_{i}^{n, l, \sigma}=\Psi_{n, l, \sigma}^{-d}+D_{i} \Psi_{n, l, \sigma}^{d},
$$

where the irreducible states $i=S$ and $A$, while the permutation coefficients $D_{S}=-D_{A}=1$. The shifted single dot wave functions acquire a phase:

$$
\Psi_{n, l, \sigma}^{ \pm d}(x, y)=\Psi_{n, l, \sigma}\left(x \pm l_{0} d, y\right) e^{ \pm i d l_{0} \theta y / l_{B}^{2}},
$$

depending on which dot they are located.

The double dot energy spectrum of $H_{0}$ as a function of magnetic field is shown in Fig. 7 for the interdot distance of $50 \mathrm{~nm}$. Indicated are two crossings and one anticrossing induced by magnetic field. States $\Gamma_{1}$ and $\Gamma_{2}$ (notation from the $B=0$ case) are not coupled because they have opposite spins. The second crossing is between $\Gamma_{2}$ and $\Gamma_{3}$, which behave differently under $I$. An example of anticrossing induced by magnetic field is between $\Gamma_{2}$ and $\Gamma_{4}$, which are both antisymmetric under $I$.

In analogy with Eq. (21) we derive analytical expressions for the energies of the lowest symmetric and antisymmetric state in the presence of magnetic field using the LCSDO approximation: 


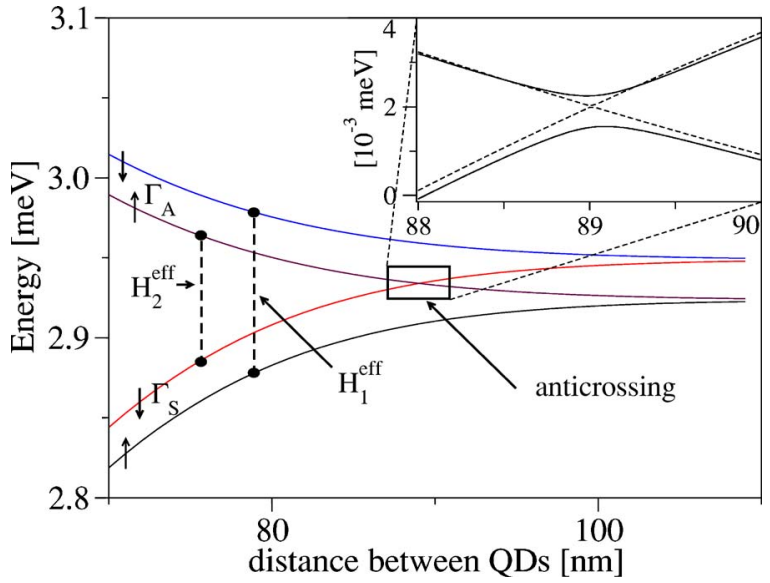

FIG. 8. (Color online) Calculated energies of the four lowest states of Hamiltonian $H_{0}+H_{Z}$ at $B=1 \mathrm{~T}$. The vertical dashed lines indicated doublets in the effective Hamiltonian. The inset displays the anticrossing at $89 \mathrm{~nm}$ due to $H_{D}$. Dashed lines are energies of $H_{0}+H_{Z}$, solid lines of $H_{0}+H_{Z}+H_{D}$.

$$
\begin{aligned}
E_{S}^{(0)}= & \frac{2 E_{0}}{\eta^{2}}\left(\frac{1+\left[1-d \eta\left(1-\theta^{2}\right) / \sqrt{\pi}\right] e^{-(d \eta)^{2}\left(1+\theta^{2}\right)}}{1+e^{-(d \eta)^{2}\left(1+\theta^{2}\right)}}\right. \\
& \left.-\frac{d \eta\left(1-\theta^{2}\right)\left[e^{-(d \eta)^{2}} / \sqrt{\pi}-d \eta \operatorname{Erfc}(d \eta)\right]}{1+e^{-(d \eta)^{2}\left(1+\theta^{2}\right)}}\right), \\
E_{A}^{(0)}= & \frac{2 E_{0}}{\eta^{2}}\left(\frac{1-\left[1-d \eta\left(1-\theta^{2}\right) / \sqrt{\pi}\right] e^{-(d \eta)^{2}\left(1+\theta^{2}\right)}}{1-e^{-(d \eta)^{2}\left(1+\theta^{2}\right)}}\right. \\
& -\frac{d \eta\left(1-\theta^{2}\right)\left[e^{-(d \eta)^{2}} / \sqrt{\pi}-d \eta \operatorname{Erfc}(d \eta)\right]}{\left.1-e^{-(d \eta)^{2}\left(1+\theta^{2}\right)}\right) .}
\end{aligned}
$$

Here $\eta=l_{0} / l_{B}=\left(1-\theta^{2}\right)^{-1 / 4}$. In the limit $d \rightarrow \infty$, we can then deduce the tunneling energy in the leading order to be

$$
T^{(0)}=E_{0} \frac{2}{\sqrt{\pi}}\left(1-\theta^{2}\right)^{5 / 4} e^{-d^{2}\left(1+\theta^{2}\right) / \sqrt{1-\theta^{2}}} .
$$

If $\theta=0$, the above expressions reduce to Eqs. (21) and (22). On the other hand, if $B \rightarrow \infty$, then $T^{(0)} \sim B^{-5 / 2} e^{-B / B_{0}}$.

At a finite magnetic field we have also a new term in the Hamiltonian, the Zeeman term. Since it commutes with $H_{0}$ the only consequence of this term is a shift of the energy of the states by a value $\sigma \mu_{B} B$ according to their spin $\sigma$. Therefore it is this term that breaks the Kramer's degeneracy and introduces crossings of the states with opposite spin. An example of this can be seen in Fig. 8, where we plot energies of the four lowest states in the region where the Zeeman shift is comparable to the energy differences of the considered states.

\section{Effective spin-orbit Hamiltonian}

We now study the influence of spin-orbit coupling on the spectrum of double dots in a finite magnetic field. We will see that spin-orbit terms lead to new spin hot spots even at magnetic fields of the order of $1 \mathrm{~T}$, and that linear spin-orbit terms will influence tunneling in the second order.

Although the presence of the Zeeman term complicates the analysis of the perturbation theory using operators $H^{o p}$, one can still apply the previously developed formalism if the Zeeman term is treated as a part of perturbation. (For a harmonic potential describing single dots, operators $H_{\text {lin }}^{o p}$ have been derived ${ }^{56}$ for the case of finite magnetic field, so that the Zeeman term can be included into $H_{0}$.) Up to the second order in the perturbation couplings (being now $\alpha_{S O}$ and $\alpha_{Z}$ ), there is no coupled Zeeman-spin-orbit term. This means that in the effective Hamiltonians $H^{\text {eff }}$ that we already derived for the case of zero magnetic field, the Zeeman term appears as a shift of the energies on the diagonal without bringing any new couplings (nondiagonal terms). But an important consequence is that the shift can change the number of states we have to include into the basis where the effective Hamiltonian acts, because their energy difference to the considered state is comparable to the spin-orbit coupling.

First, in analogy with Eq. (28), if the energy of a state is far enough from the others, we can consider the basis to consist of one term only and the spin-orbit correction to the energy of state $|i\rangle$ is

$$
\delta E_{i}^{S O}=-E_{0} \frac{\alpha_{D}^{2}}{2}\left(1-\sigma \overline{L_{z}}\right)-E_{0} \frac{\alpha_{B R}^{2}}{2}\left(1+\sigma \overline{L_{z}}\right)+\overline{\left[H_{D}^{o p}, H_{D 3}\right]},
$$

where the averaging means the expectation value in the state $|i\rangle$ and $\sigma$ is the spin of the state. Since the presence of magnetic field lowers the symmetry, the last commutator, [Eq. (15)], can not be simplified according to the symmetry as was the case before in Eq. (29), and, more important, we no longer have $\overline{L_{z}}=0$. As a result, there are now corrections to the tunneling that are of the second order in the linear spinorbit couplings. These corrections depend on $\alpha_{-}^{(2)} \equiv \alpha_{D}^{2}$ $-\alpha_{B R}^{2}$

Second, we look how the energies of the four lowest states are changed, using again the example of the linear Dresselhaus term. They are plotted in Fig. 8. Here in the main figure one can see the shift caused by the Zeeman term and the anticrossing induced by the spin-orbit coupling is magnified in the inset. The anticrossing states are $\Gamma_{S}^{\downarrow}$ and $\Gamma_{A}^{\uparrow}$. In the case of zero magnetic field we described each of the four basis states by Eq. (28). Now, in principle, we have to describe them by a $4 \times 4$ effective Hamiltonian Eq. (25). Due to symmetry we can simplify this Hamiltonian into two 2 $\times 2$ Hamiltonians, $H_{1}^{\text {eff }}, H_{2}^{\text {eff }}$, acting in the bases $\Gamma_{S}^{\uparrow}, \Gamma_{A}^{\downarrow}$ and $\Gamma_{S}^{\downarrow}, \Gamma_{A}^{\uparrow}$, respectively. The four components of the effective Hamiltonian matrix are

$$
\begin{aligned}
& \left(H^{\mathrm{eff}}\right)_{11}=E_{S}^{(0)}-E_{0} \frac{\alpha_{D}^{2}}{2}\left[1-\sigma\left(L_{z}\right)_{11}\right]-\sigma \mu_{B} B-R_{11}, \\
& \left(H^{\mathrm{eff}}\right)_{22}=E_{A}^{(0)}-E_{0} \frac{\alpha_{D}^{2}}{2}\left[1+\sigma\left(L_{z}\right)_{22}\right]+\sigma \mu_{B} B+R_{11},
\end{aligned}
$$




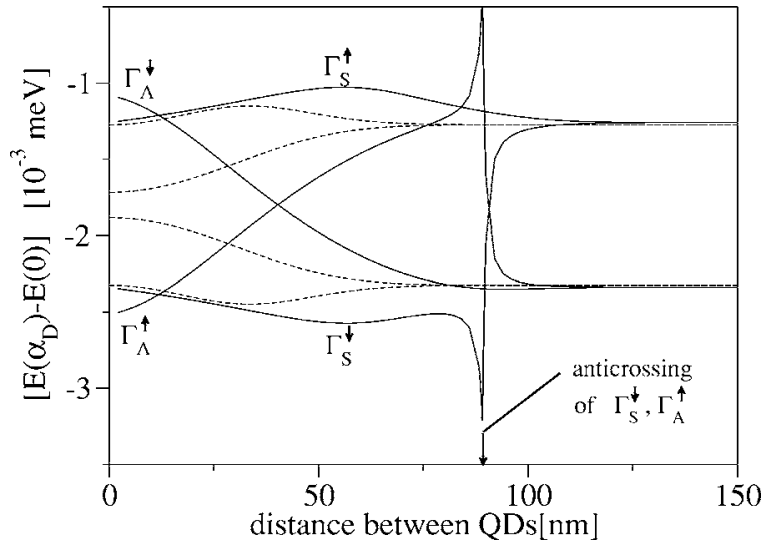

FIG. 9. Calculated corrections to the energies of the four lowest states due to the linear Dresselhaus term $H_{D}$, at $B=1 \mathrm{~T}$. Solid lines are numerical data; dashed lines are analytical expressions computed by Eq. (35) using the LCSDO approximation for the states.

$$
\left(H^{\text {eff }}\right)_{12}=\left(H^{\text {eff }}\right)_{21}^{\dagger}=\left(H_{D}\right)_{12}
$$

where $\sigma=1$ for $H_{1}^{\text {eff }}$ and $\sigma=-1$ for $H_{2}^{\text {eff }}$, while indices 1,2 denote the first and the second term in the corresponding basis. Comparing to the case of zero magnetic field the Zeeman term increases the difference of the diagonal elements in $H_{1}^{\text {eff }}$ and decreases them in $H_{2}^{\text {eff }}$. The ground and the fourth excited states which are described by $H_{1}^{\text {eff }}$ stay isolated, and we can do the perturbative diagonalization to get rid of the off-diagonals. The energy of the two states is then up to the second order in the spin-orbit coupling accurately described by Eq. (35). Concerning the states $\Gamma_{S}^{\downarrow}$ and $\Gamma_{A}^{\uparrow}$, there is a region in the interdot distance of a few nanometers, where the two states must be described by the two dimensional $H_{2}^{\text {eff }}$ to account for the anti-crossing, which is caused by the the matrix element $\left\langle\Gamma_{S}^{\downarrow}\left|H_{D}\right| \Gamma_{A}^{\uparrow}\right\rangle$. LCSDO gives for this element a result correct only in the order of magnitude. This is because even in the limit $d \rightarrow \infty$ this matrix element is of the same order as the neglected coefficients $c(n, l)$ in the LCSDO approximation, Eq. (20).

The spin-orbit corrections to the energies from $H_{D}$ for the four lowest states as functions of the interdot distance are in Fig. 9. Also shown are analytical values computed by Eq. (35), that is, ignoring anticrossing. The scale implies that the corrections are of the second order in $\alpha_{D}$ and for the states $\Gamma_{S}^{\downarrow}$ and $\Gamma_{A}^{\uparrow}$ are enhanced in the anticrossing region.

\section{E. Spin-orbit corrections to the effective $g$ factor and tunneling frequency}

We next analyze spin-orbit corrections to the $g$ factor, $\delta g \equiv\left[\delta E\left(\Gamma_{S}^{\downarrow}\right)-\delta E\left(\Gamma_{S}^{\uparrow}\right)\right] / \mu_{B} B$, that characterizes the energy cost of a spin flip in the ground state, or the frequency of a spin precession. Another kind of oscillation is electron tunneling, when electron oscillates between the left and the right dot without changing its spin. The frequency of this oscillation, $T / h$, is given by the energy difference $T^{\uparrow}=\left[E\left(\Gamma_{A}^{\uparrow}\right)-E\left(\Gamma_{S}^{\uparrow}\right)\right] / 2$. Corrections to this energy difference induced by the spin-orbit interaction are denoted by $\delta T^{\sigma, S O}$.
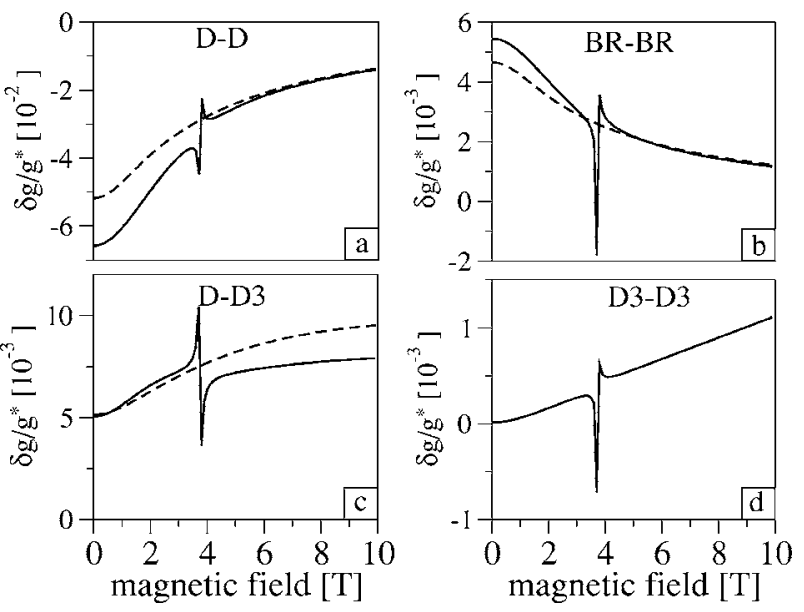

FIG. 10. Calculated spin-orbit corrections to the effective $g$ factor (relative to the conduction band value $g^{*}$ ) as functions of magnetic field. The distance between the dots is $50 \mathrm{~nm}$. Solid lines are numerical data; dashed lines are analytical values computed using Eq. (35). Contributions come from linear spin-orbit terms $(a, b)$, the mixed Dresselhaus correction from $H_{D}$ and $H_{D 3}$ (c) and the cubic Dresselhaus $H_{D 3}$ correction (d).

First, we take a look at the spin-orbit corrections to the $g$ factor. Contributions in the second order of the spin-orbit couplings are shown in Fig. 10, as functions of magnetic field at a constant interdot distance. The spin-orbit contribution to the $g$ factor in the double dot case has the same qualitative dependence on the magnetic field as in the single dot case (see discussion to Fig. 2). However, at finite interdot distances, there is an enhancing effect on the spin-orbit contributions. This can be seen in Fig. 9, where at a certain magnetic field, the spin-orbit contribution to the $g$ factor is enhanced for a finite $d$ compared to the case of $d=0(\infty)$. We found numerically that the enhancement can be up to $50 \%$ of the value of the correction in $d=0$ at magnetic fields of the order of $1 \mathrm{~T}$.

At the anticrossing the spin-orbit contributions show cusps. At magnetic fields bellow the anticrossing, the dominant spin-orbit contribution is $D-D$ which reduces the conduction band $g$ factor by several percent. Contributions $D-D 3$ and $B R-B R$ are one order of magnitude smaller. Using Eq. (35), which means that we ignore anticrossing effects, we get for the contribution from the linear spin-orbit terms

$$
\delta g_{\text {lin-lin }}=-\frac{E_{0}}{\mu_{B} B} \alpha_{-}^{(2)} \overline{L_{z}},
$$

where, in the limit $d \rightarrow \infty,\left[\eta=l_{0} / l_{B}=\left(1-\theta^{2}\right)^{-1 / 4}\right]$,

$$
\overline{L_{z}} \approx \theta\left[1+(d / \eta)^{2} e^{-(d \eta)^{2}\left(1+\theta^{2}\right)}\right] .
$$

From Fig. 10 one can see that the analytical result agrees with numerics.

Finally, we look at the tunneling energy in the presence of both magnetic field and spin-orbit couplings. The spin-orbit corrections, as a function of magnetic field, are shown in Fig. 11. At zero magnetic field there is no contribution from the linear terms (result of Sec. IV B) and the dominant contribu- 

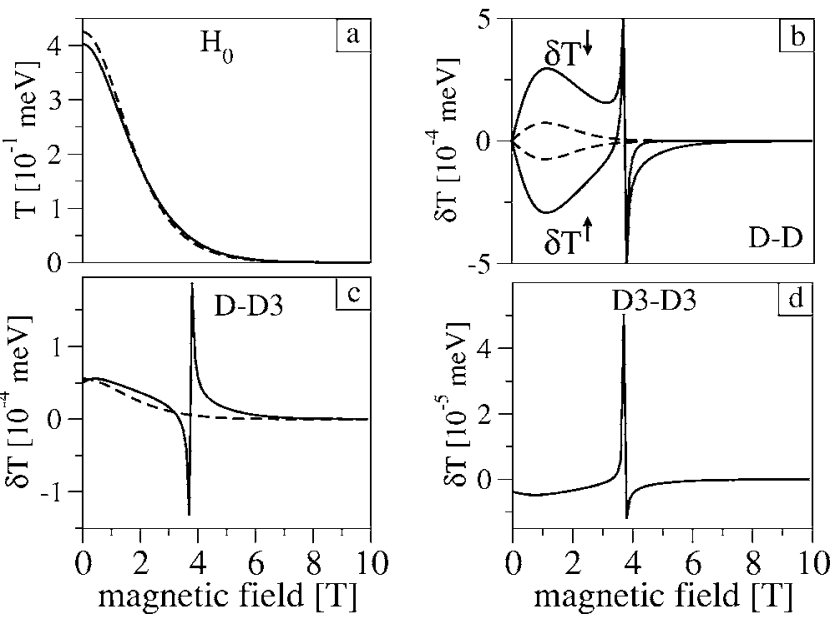

FIG. 11. Calculated spin-orbit corrections to the tunneling energy $T$ as a function of magnetic field. The interdot distance is $50 \mathrm{~nm}$. Solid lines are numerical data; dashed lines are analytical expressions computed by Eq. (35). (a) Tunneling without spin-orbit interactions present. (b) Contribution to the tunneling from linear Dresselhaus for both spin up and down. (c) The mixed linear-cubic and (d) cubic-cubic Dresselhaus contributions.

tion is $D-D 3$. Similarly as $T^{(0)}$, the corrections decay exponentially with increasing magnetic field. Anticrossing strongly influences the tunneling energy. Using LCSDO for $d \rightarrow \infty$ we obtain in the leading order

$$
\delta T^{\uparrow, \text { lin }}=-\left(E_{0} / 2\right) \alpha^{(2)} \theta(d / \eta)^{2} e^{-(d \eta)^{2}\left(1+\theta^{2}\right)} .
$$

This analytical formula underestimates the corrections from the linear spin-orbit terms by a factor of $\sim 3$. Nevertheless the analytical expression for $D-D 3$ is reasonably good. In the magnetic field below anticrossing, the relative change of the tunneling energy stemming from the spin-orbit terms is of order $10^{-3}$. In the inset $b$, there are contributions to the tunneling from the linear Dresselhaus term for both spin states. The importance of their difference will be explained in the next section.

\section{F. Tunneling Hamiltonian}

We now use our results to describe the influence of the spin-orbit interaction on the lower part of the spectrum. We restrict our Hilbert space on the four lowest states $\Gamma_{S(A)}^{\sigma}$, the eigenstates of the total double dot Hamiltonian. Because of the transformation Eq. (30) these four states have neither definite symmetry with respect to inversion $I$, nor a definite spin in the $\mathbf{z}$ direction. In this section we will denote them as spin "up" and spin "down" states. For a description of a transport through the double dot it is useful to define the following left and right localized functions:

$$
L^{\sigma}\left(R^{\sigma}\right)=\frac{1}{\sqrt{2}}\left(\Gamma_{S}^{\sigma} \pm \Gamma_{A}^{\sigma}\right),
$$

where plus and minus holds for $L$ and $R$, respectively. In the limit $d \rightarrow \infty$ these functions converge to single dot solutions localized in the left and right dot.
The effective Hamiltonian of our system in the second quantization formalism is

$$
H=\sum_{\sigma=\uparrow, \downarrow} E^{\sigma}\left(n_{L \sigma}+n_{R \sigma}\right)-T^{\sigma}\left(a_{L \sigma}^{\dagger} a_{R \sigma}+a_{R \sigma}^{\dagger} a_{L \sigma}\right),
$$

where $E^{\sigma}=\left(E_{S}^{\sigma}+E_{A}^{\sigma}\right) / 2, T^{\sigma}=\left(E_{A}^{\sigma}-E_{S}^{\sigma}\right) / 2$, while $a^{\dagger}$, and $a$ are creation and annihilation operators, and $n=a^{\dagger} a$. We can get both localized and spin-pure states if we diagonalize $\sigma_{z}$ in a chosen basis. For example, taking $L^{\uparrow}$ and $L^{\downarrow}$, we get $L^{\text {pure }}$ $\sim\left(L^{\uparrow}+o L^{\downarrow}\right)$ and $L^{\text {pure } \downarrow} \sim\left(L^{\downarrow}-o^{+} L^{\uparrow}\right)$, up to normalization $\left(1-|o|^{2} / 2\right)$. That is, the left pure spin state is a linear superposition of both left spin "up" and left spin "down" states. The coefficient $o$ is proportional to $\alpha_{S O}$.

In the following we are interested in the time evolution of localized states given by Hamiltonian Eq. (41). First, we note that due to the nondiagonal terms, the electron which is in a localized state will tunnel into the other localized state after the tunneling time $t_{\text {tun }}^{\sigma}=h / 2 T^{\sigma}$, resulting in coherent oscillations. For our parameters $t_{\text {tun }} \approx 1 \mathrm{ps}$. In the Hamiltonian there is no mixing between spin "up" and spin "down" states. However, there will be mixing (or spin-flip) if we work with localized pure spin states. Electrons appearing originally in $L^{\text {pure } \uparrow}$ will, after the tunneling time $t_{\text {tun }}^{\uparrow}$, contain $R^{\text {pure } \downarrow}$ with the probability amplitude

$$
c=i o \pi\left(T^{\downarrow}-T^{\uparrow}\right) / 4 T^{\uparrow},
$$

assuming that the difference in $T$ for different spins is much smaller that $T$ itself.

In the case of zero magnetic field, because of Kramer's degeneracy, the tunneling frequencies are the same for both spin orientations. Then whatever is the initial combination of spin "up" and "down" (let it be, for example, $\left.L^{\text {pure } \uparrow}\right)$, during the time evolution (oscillations) there will be no relative change in the coefficients in this linear combination. Therefore spin-orbit coupling does not lead to spin-flipping and $c=0$ in Eq. (42).

In a finite magnetic field, the tunneling frequency for spin "up" and "down" are different. The difference is caused by spin-orbit terms only, and is of order $\alpha_{-}^{(2)}=\alpha_{D}^{2}-\alpha_{B R}^{2}$. Equation (35) shows that the contribution to $T^{\uparrow}$ from the linear spin-orbit terms is opposite that of $T^{\downarrow}$ and therefore their difference is twice the expression in Eq. (39), which is of the second order in spin-orbit couplings. This can be seen in Fig. 11(b). We conclude, that spin-flip during tunneling induced by spin-orbit coupling is proportional to the third power in spin-orbit couplings and depends linearly on the magnetic field if the magnetic field is small $\left(c \sim \alpha_{\operatorname{lin}}^{3} \theta\right)$.

The different tunneling frequency can be exploited for separation of different spin states in a homogeneous magnetic field. Starting with some combination of "up" and "down" states localized in one dot, after time $t_{\text {sep }}=h / 2\left(T^{\uparrow}\right.$ $\left.-T^{\downarrow}\right)=t_{\text {tun }} T^{\uparrow} /\left(T^{\uparrow}-T^{\downarrow}\right)$ the part with spin "up" will be localized in one, and the spin "down" in the other dot. From Fig. 11 one can see that about $10^{3}$ coherent oscillations are needed to get the spatial spin separation. Although the relaxation and decoherence times for spin-flip in quantum dots (of the order of $1 \mathrm{~ms}-1 \mu \mathrm{s}^{14,19}$ ) are much longer than the separation time; for this scheme to work we need to retain also 
the charge coherence during the separation. The charge relaxation time is comparable to the separation time $(0.1 \mathrm{~ns}$ in Ref. 57). We note that the separated states will not be pure spin states, but will contain a small (linearly proportional to $\alpha_{S O}$ ) admixture of opposite pure spin states.

\section{CONCLUSIONS}

We have performed numerically exact calculation of the spectrum of a single electron localized by a confining potential in single and double GaAs quantum dots. We have studied the influence of the spin-orbit terms, namely, the Bychkov-Rashba and the linear and cubic Dresselhaus terms, on the energy spectrum. In the single dot case we have elaborated on previous results and have shown that the spin-orbit interaction has three principal effects on the spectrum: first, the interaction shifts the energy by a value proportional to the second order in the spin-orbit couplings, second, it lifts the degeneracy at zero magnetic field, and, third, the Bychkov-Rashba term gives rise to spin hot spots at finite magnetic fields.

In the double dot case we have addressed the symmetries of the Hamiltonian. For zero magnetic field without spinorbit terms we have constructed the correlation diagram, between single and double dot states of the spectrum. We have used properly symmetrized linear combination of shifted single dot solutions as an approximation for a double dot solution and found that for the four lowest states it gives a good approximation for the energy. As for the contributions to the energy from the linear spin-orbit terms, we have found that in zero magnetic field a typical feature of a double dot is a uniform shift of the energy proportional to the second order in the coupling strengths without any dependence on the interdot distance. This is true also if the potential has lower (or none) symmetry (for example biased dots). Therefore, in zero magnetic field, there is no influence on the tunneling frequency up to the second order in the linear spin-orbit couplings and the dominant contribution comes from the mixed linear and cubic Dresselhaus second order term. We found, that spin hot spots in zero magnetic field exist in the double dot, but are solely due to the cubic Dresselhaus term. This means also, that for our potential, for the cubic Dresselhaus term there can not exist a unitary transformation to eliminate its contribution in the first order.

The effective $g$ factor, on the other hand, is influenced by the second order linear spin-orbit couplings even at $B \sim 0$, so the dominant contribution here is the linear Dresselhaus term for GaAs. In finite magnetic fields the uniform shift does not hold any more and there is a contribution to the tunneling frequency in the second order of the linear spin-orbit couplings. We have derived an effective Hamiltonian, using Löwdin's perturbation theory, with which analytical results up to the second order in perturbations (Zeeman and spinorbit terms with the exception of cubic Dresselhaus-cubic Dresselhaus contribution) can be obtained provided one has exact solutions of the double dot Hamiltonian without Zeeman and spin-orbit terms. From this effective Hamiltonian we have derived the uniform shift in zero magnetic field. In a finite magnetic field we used linear combinations of single dot solutions to obtain analytical expressions for the spin-orbit contributions to the energy for the four lowest states. We have analyzed them as functions of the interdot distance and magnetic field and compared them with exact numerical values. The spin-orbit relative contribution to the $g$ factor and the tunneling frequency is of the order of $\sim 10^{-2}$ and $\sim 10^{-3}$, respectively. Due to the degeneracy of the energy spectrum at large interdot distance the spin hot spots exist also at smaller magnetic fields compared to the single dot case.

In this paper we had positioned the vector connecting the double dots to be parallel to the crystallographic $\mathbf{x}$ axis. Our results hold if the orientation of the dots is arbitrary. If we stay in the coordinates system of the crystallographic axes, expressions for the spin-orbit contribution to the energy achieved by using $H^{o p}$ stay the same. The symmetry group of the double dot Hamiltonian without spin-orbit terms is the same as before $-\mathrm{C}_{2 v}$, only the axes of reflections are rotated. The symmetry properties of the spin-orbit Hamiltonians with respect to reflections given by these axes are the same (they still transform as $x \oplus y$ ). Therefore all the considerations based on $H^{o p}$ and symmetry will hold. The linear spin-orbit terms appear in the effective Hamiltonians through matrix elements of $L_{z}$ operator, which is invariant to rotations in the $x y$ plane.

As an application of our results we have constructed an effective Hamiltonian acting in a restricted Hilbert space of four states-electron localized on either dots with up and down spins; (these are effective spins in the presence of spinorbit coupling). For these effective spins there is only spinconserving tunneling between the localized states, no spinflip tunneling. In zero magnetic field the spin-orbit interaction does not significantly influence the tunneling frequency, nor does it imply spin-flip tunneling even for Pauli spin states. In finite magnetic fields the tunneling frequency is spin dependent; the difference being of second order in linear spin-orbit terms. This leads to a spin flip amplitude for Pauli spins proportional to the third power in spin-orbit couplings (it is linear in magnetic field). We propose to use this difference of the tunnelings to spatially separate electron spin in homogeneous magnetic fields.

\section{ACKNOWLEDGMENTS}

This work was supported by the US ONR. We thank Ulrich Hohenester for useful discussions. 
${ }^{1}$ S. Datta and B. Das, Appl. Phys. Lett. 56, 665 (1990).

${ }^{2}$ J. Nitta, T. Akazaki, H. Takayanagi, and T. Enoki, Phys. Rev. Lett. 78, 1335 (1997).

${ }^{3}$ T. Koga, J. Nitta, T. Akazaki, and H. Takayanagi, Phys. Rev. Lett. 89, 046801 (2002).

${ }^{4}$ G. Dresselhaus, Phys. Rev. 100, 580 (1955).

${ }^{5}$ Y. A. Bychkov and E. I. Rashba, J. Phys. C 17, 6039 (1984).

${ }^{6}$ E. I. Rashba, Fiz. Tverd. Tela (Leningrad) 2, 1224 (1960), [Sov. Phys. Solid State 2, 1109 (1960)].

${ }^{7}$ I. Žutić, J. Fabian, and S. Das Sarma, Rev. Mod. Phys. 76, 323 (2004).

${ }^{8}$ D. Loss and D. P. DiVincenzo, Phys. Rev. A 57, 120 (1998).

${ }^{9}$ G. Burkard, D. Loss, and D. P. DiVincenzo, Phys. Rev. B 59, 2070 (1999).

${ }^{10}$ X. Hu and S. Das Sarma, Phys. Rev. A 61, 062301 (2000).

${ }^{11}$ J. Fabian and U. Hohenester, cond-mat/0412229 (2004) (unpublished).

${ }^{12}$ G. Burkard and D. Loss, Phys. Rev. Lett. 88, 047903 (2002).

${ }^{13}$ D. Stepanenko, N. E. Bonesteel, D. P. DiVincenzo, G. Burkard, and D. Loss, Phys. Rev. B 68, 115306 (2003).

${ }^{14}$ A. V. Khaetskii and Y. V. Nazarov, Phys. Rev. B 64, 125316 (2001).

${ }^{15}$ C. F. Destefani and S. E. Ulloa, Phys. Rev. B (to be published).

${ }^{16}$ L. M. Woods, T. L. Reinecke, and Y. Lyanda-Geller, Phys. Rev. B 66, 161318(R) (2002).

${ }^{17}$ J. L. Cheng, M. W. Wu, and C. Lü, Phys. Rev. B 69, 115318 (2004).

${ }^{18}$ V. N. Golovach, A. Khaetskii, and D. Loss, Phys. Rev. Lett. 93, 16601 (2004).

${ }^{19}$ D. V. Bulaev and D. Loss, Phys. Rev. B 71, 205324 (2005).

${ }^{20}$ J. R. Petta, A. C. Johnson, C. M. Markus, M. P. Hanson, and A. C. Gossard, Phys. Rev. Lett. 93, 186802 (2004).

${ }^{21}$ T. Hayashi, T. Fujisawa, H. D. Cheong, Y. H. Jeong, and Y. Hirayama, Phys. Rev. Lett. 91, 226804 (2003).

${ }^{22}$ W. G. van der Wiel, S. De Franceschi, J. M. Elzerman, T. Fujisawa, S. Tarucha, and L. P. Kouwenhoven, Rev. Mod. Phys. 75, 1 (2003).

${ }^{23}$ J. R. Petta, A. C. Johnson, A. Jacoby, C. M. Marcus, M. P. Hanson, and A. C. Gossard, Phys. Rev. B (to be published).

${ }^{24}$ R. Hanson, B. Witkamp, L. M. K. Vandersypen, L. H. Willems van Beveren, J. M. Elzerman, and L. P. Kouwenhoven, Phys. Rev. Lett. 91, 196802 (2003).

${ }^{25}$ R. Hanson, L. H. Willems van Beveren, I. T. Vink, J. M. Elzerman, W. J. M. Naber, F. H. L. Koppens, L. P. Kouwenhoven, and L. M. K. Vandersypen, Phys. Rev. Lett. 94, 196802 (2005).

${ }^{26}$ A. C. Johnson, J. R. Petta, J. M. Taylor, A. Yacobi, M. D. Lukin, C. M. Marcus, M. P. Hanson, and A. C. Gossard, Nature (London) 435, 925 (2005).

${ }^{27}$ O. Voskoboynikov, C. P. Lee, and O. Tretyak, Phys. Rev. B 63, 165306 (2001).
${ }^{28}$ M. Governale, Phys. Rev. Lett. 89, 206802 (2002).

${ }^{29}$ R. de Sousa and S. Das Sarma, Phys. Rev. B 68, 155330 (2003).

${ }^{30}$ M. Valin-Rodriguez, A. Puente, and L. Serra, Phys. Rev. B 69, 153308 (2004).

${ }^{31}$ C. F. Destefani, S. E. Ulloa, and G. E. Marques, Phys. Rev. B 70, 205315 (2004).

${ }^{32}$ C. F. Destefani, S. E. Ulloa, and G. E. Marques, Phys. Rev. B 69, 125302 (2004).

${ }^{33}$ W. H. Kuan, C. S. Tang, and W. Xu, J. Appl. Phys. 95, 6368 (2004).

${ }^{34}$ E. Tsitsishvili, G. S. Lozano, and A. O. Gogolin, Phys. Rev. B 70, 115316 (2004).

${ }^{35}$ O. Voskoboynikov, O. Bauga, C. P. Lee, and O. Tretyak, J. Appl. Phys. 94, 5891 (2003).

${ }^{36}$ E. N. Bulgakov and A. F. Sadreev, JETP Lett. 73, 505 (2001).

${ }^{37}$ P. Pfeffer and W. Zawadzki, Phys. Rev. B 59, R5312 (1999).

${ }^{38}$ C. F. Destefani and S. E. Ulloa, Phys. Rev. B 71, 161303(R) (2005).

${ }^{39}$ J. Fabian and S. Das Sarma, Phys. Rev. Lett. 81, 5624 (1998).

${ }^{40} \mathrm{H}$. Engel and D. Loss, Science 309, 586 (2005).

${ }^{41}$ P. Recher, E. V. Sukhorukov, and D. Loss, Phys. Rev. Lett. 85, 1962 (2000).

${ }^{42}$ A. D. Greentree, A. R. Hamilton, L. C. L. Hollenberg, and R. G. Clark, Phys. Rev. B 71, 113310 (2005).

${ }^{43}$ R. Ionicioiu and A. E. Popescu, New J. Phys. 7, 120 (2005).

${ }^{44}$ Elzermann, R. Hanson, L. H. Williams van Beveren, B. Witkamp, L. M. K. Vandersypen, and L. P. Kouwenhoven, Nature (London) 430, 431 (2002).

${ }^{45}$ M. Dyakonov and V. Kachorovskii, Fiz. Tekh. Poluprovodn. (S.Peterburg) 20, 178 (1986), [Sov. Phys. Semicond. 20, 110 (1986)].

${ }^{46}$ J. Miller, D. Zumbuhl, C. Marcus, Y. Lyanda-Geller, D. Goldhaber-Gordon, K. Campman, and A. Gossard, Phys. Rev. Lett. 90, 76807 (2003).

${ }^{47}$ W. Knap, C. Skierbiszewski, A. Zduniak, E. Litwin Staszewska, D. Bertho, F. Kobbi, J. L. Robert, G. E. Pikus, F. G. Pikus, S. V. Iordanskii, V. Mosser, K. Zekentes, and Yu. B. Lyanda-Geller, Phys. Rev. B 53, 3912 (1996).

${ }^{48}$ V. Fock, Z. Phys. 47, 446 (1928).

${ }^{49}$ C. G. Darwin, Proc. Cambridge Philos. Soc. 27, 86 (1931).

${ }^{50}$ I. Aleiner and V. I. Fălko, Phys. Rev. Lett. 87, 256801 (2001).

${ }^{51}$ J. Fabian and S. Das Sarma, Phys. Rev. Lett. 83, 1211 (1999).

${ }^{52}$ M. Braun and U. Rossler, J. Phys. C 18, 3365 (1985).

${ }^{53}$ M. Valín-Rodriguez, A. Puente, L. Serra, and E. Lipparini, Phys. Rev. B 66, 235322 (2002).

${ }^{54}$ G. F. Koster, Solid State Physics, edited by F. Seitz and D. Turnbull (Academic Press, New York, 1957), Vol. 5, p. 173.

${ }^{55}$ P. Löwdin, J. Chem. Phys. 19, 1396 (1951).

${ }^{56}$ M. Valin-Rodriguez, Phys. Rev. B 70, 033306 (2004).

${ }^{57}$ V. N. Stavrou and X. Hu, Phys. Rev. B 72, 075362 (2005). 